\title{
Many-Body Quantum Lock-In Amplifier
}

\author{
Min Zhuang, ${ }^{1}$ Jiahao Huang, ${ }^{1, *}$ and Chaohong Lee ${ }^{1,2, \dagger}$ \\ ${ }^{1}$ Guangdong Provincial Key Laboratory of Quantum Metrology and Sensing \& School of Physics and Astronomy, \\ Sun Yat-Sen University (Zhuhai Campus), Zhuhai, 519082, China \\ ${ }^{2}$ State Key Laboratory of Optoelectronic Materials and Technologies, Sun Yat-Sen University (Guangzhou \\ Campus), Guangzhou, 510275, China
}

(Received 9 October 2020; revised 3 April 2021; accepted 7 September 2021; published 26 October 2021)

Achieving high-precision detection of time-dependent signals within noise is a ubiquitous issue in physics and a critical task in metrology. Lock-in amplifiers are detectors that can extract alternating signals within extreme noise via a known carrier frequency. Here we present a protocol for achieving an entanglement-enhanced lock-in amplifier via use of many-body multipulse quantum interferometry. The many-body quantum lock-in amplifier is implemented by application of a periodic multi- $\pi$-pulse sequence during the interrogation. Our analytic results show that, by our choosing suitable input states and readout operations, the frequency and amplitude of an unknown alternating field can be simultaneously extracted via population measurements. The lock-in point can be determined via the symmetry of the signal during a single interrogation time or the time-averaged signals for multiple interrogation times. We find that the measurement signal at the lock-in point is independent of the interrogation time. In particular, if we input spin cat states and apply interaction-based readout operations, the measurement precisions for frequency and amplitude can both approach the Heisenberg limit. Moreover, our many-body quantum amplifier is also robust with regard to extreme stochastic noise. Our study paves a new way for measuring time-dependent signals with many-body quantum systems, and provides a feasible method for achieving Heisenberg-limited detection of alternating signals.

DOI: 10.1103/PRXQuantum.2.040317

\section{INTRODUCTION}

Detecting time-dependent alternating signals with high precision is an important problem in fundamental physics and a critical task in metrology [1-6]. Generally, the target signal is submerged in stochastic noise, in which the target signal and the stochastic noise couple to the probe through the same physical channel. To obtain a high signal-tonoise ratio, one has to choose an optimal trade-off between decreasing the effect of noise and enhancing the response to the target signal. Usually, a lock-in amplifier has been used as a high-efficiency classical detector to extract a signal from noise with a high signal-to-noise ratio. The lock-in amplifier selects the signal with a specific frequency and phase from a mixture of mostly unwanted frequencies.

\footnotetext{
*hjiahao@mail2.sysu.edu.cn, eqjiahao@gmail.com

$\dagger$ †ichaoh2@mail.sysu.edu.cn, chleecn@gmail.com
}

Published by the American Physical Society under the terms of the Creative Commons Attribution 4.0 International license. Further distribution of this work must maintain attribution to the author(s) and the published article's title, journal citation, and DOI.
Dynamical decoupling (DD) methods have been extensively used to increase the signal-to-noise ratio of quantum sensors for alternating signals [7-26]. In particular, a quantum analog of the classical lock-in amplifier with a single trapped $\mathrm{Sr}^{+}$ion has been demonstrated [23], in which noncommuting manipulations are performed to decouple the quantum probe from noise, while increasing its sensitivity. Quantum lock-in techniques are readily available for other quantum probes [20-26]. However, to the best of our knowledge, all existing studies of quantum lockin amplifiers concentrate on single-particle systems. Can one achieve a quantum lock-in amplifier via a many-body quantum system?

It is well known that many-body quantum entanglement is a useful resource for increasing measurement precision [15,27-32]. For $N$ individual particles, the measurement precision scales as the standard quantum limit (SQL) (i.e., $\propto 1 / \sqrt{N}$ ). The SQL can be surpassed by using entanglement. Remarkably, entangled non-Gaussian states (ENGSs), such as spin cat states or even GreenbergerHorne-Zeilinger (GHZ) states, set a benchmark for beating the SQL in metrology. The measurement precision can be increased to the Heisenberg limit (HL) (i.e., $\propto 1 / N$ ) [33-37]. With state-of-art techniques, the ENGS state can 
be generated in ultracold atomic gases [12,32,38,38-43], trapped ions [44], and photonic systems [45]. Can one use many-body quantum entanglement to increase the measurement precision of a quantum lock-in amplifier? Furthermore, is the entanglement-enhanced lock-in amplifier robust with regard to extreme stochastic noise?

In this article, we present a general protocol for achieving a many-body quantum lock-in amplifier via quantum interferometry under a periodic multipulse sequence. Many-body quantum interferometry can be divided into three steps: state preparation, signal interrogation, and readout. To detect an unknown alternating signal, we apply a train of $\pi$ pulses with equidistant spacing in the signal interrogation stage. The modulation (i.e., the applied $\pi$-pulse sequence) is noncommuting with the signal acquisition, which is the key for achieving a quantum lock-in amplifier. For a given evolution time $T$, the pulse spacing $\tau=\pi / \omega_{e}$ is adjusted to probe the oscillating frequency $\omega$ of the target signal. At resonance $\omega_{e}=\omega$, when the signal and the modulation are locked in, the $\pi$ pulses are applied at every peak and valley of the signal and thus the accumulated phase is close to zero, while for $\omega_{e} \neq \omega$, the accumulated phase oscillates dramatically and is sensitively dependent on the detuning $\omega-\omega_{e}$. As this accumulated phase determines the half-population, one may infer the lock-in point $\omega_{e}=\omega$ by measuring the half-population versus the detuning. Meanwhile, the signal amplitude can also be extracted.

Moreover, we analytically study the measurement precisions for frequency and amplitude of an ac magnetic field. For nonentangled states, the measurement precisions only attain the SQL. Through use of entangled states, the measurement precisions can be increased to the Heisenberg limit. In particular, by use of spin cat states and suitable interaction-based readout operations [46-56], the measurement precisions for frequency and amplitude can simultaneously achieve the Heisenberg limit. Besides, our many-body quantum amplifiers are robust with regard to stochastic noise, even with highly entangled spin cat states.

This article is organized as follows. In Sec. II, we introduce our general protocol for a many-body quantum lock-in amplifier by applying a train of $\pi$ pulses. In Sec. III, we study the measurement precisions for different input states. We demonstrate how to achieve the Heisenberglimited quantum lock-in amplifier via spin cat states and discuss its robustness against stochastic noise. Finally, we give a brief summary and discussion in Sec. IV.

\section{GENERAL PROTOCOL}

In this section, we present our general protocol for achieving a many-body quantum lock-in amplifier. Classical lock-in amplifiers can extract a signal with a known carrier frequency from noise; see Fig. 1(a). The noise is mixed with the target signal (i.e., the target signal and the noise couple to the probe through the same physical channel). In essence, a lock-in amplifier receives a target signal, multiplies it by a reference signal, and performs integration over a specified time. After the integration, there is almost no contribution from any signal that is not at the same frequency as the reference signal. In analogy, a quantum lock-in amplifier with many-body quantum systems can be realized by use of quantum control techniques; see Fig. 1(b). For the classical lock-in amplifier, the mix-down process can generate the instantaneous product of the target signal and the reference signal and can be achieved with a nonlinear device. Correspondingly, the key for realizing a quantum lock-in amplifier is to find a quantum analog of the mix-down process [23]. Nonlinear dynamics of the wave function cannot be introduced directly. However, the dynamics is proportional to a product of
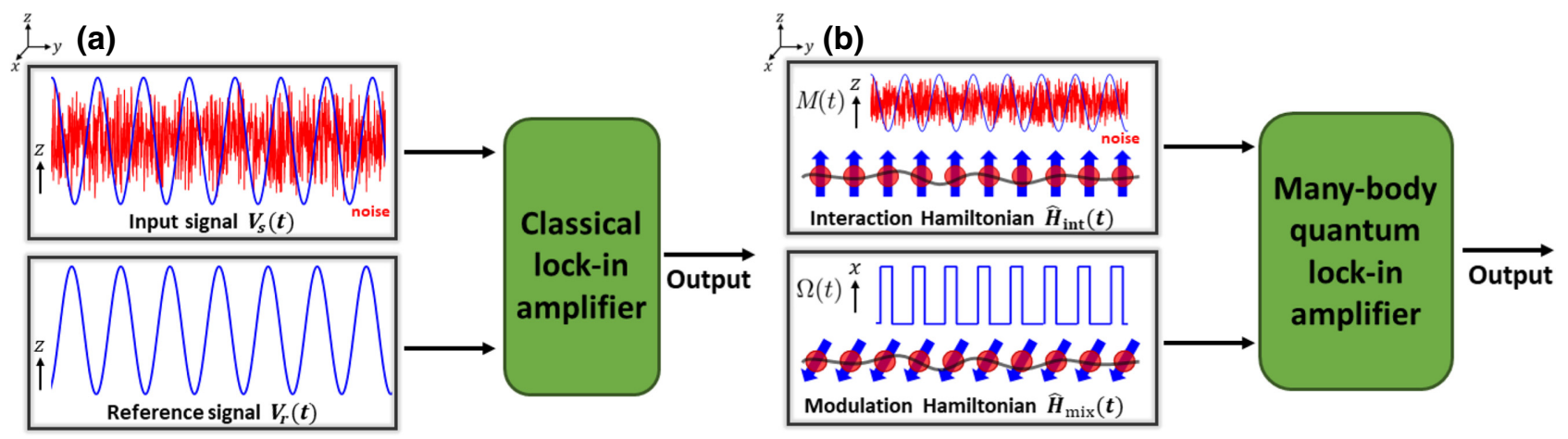

FIG. 1. A classical lock-in amplifier and a many-body quantum lock-in amplifier. (a) The classical lock-in amplifier. $V_{s}(t)$ is the target signal submerged in extreme noise. $V_{r}(t)$ is a known reference signal. Inputting the two signals through the classical lock-in amplifier, one can extract the signal $V_{S}(t)$. (b) The many-body quantum lock-in amplifier. The coupling between the probe and the signal is described by $\hat{H}_{\text {int }}=M(t) \hat{J}_{z}$, with $M(t)=S(t)+N_{o}(t)$, where $S(t)$ is the target signal and $N_{o}(t)$ is the stochastic noise. The oscillating modulation term $\hat{H}_{\text {mix }}$, which is analogous to the reference signal $V_{r}(t)$, does not commute with $\hat{H}_{\text {int }}$. Thus, the quantum probe, obeying the Hamiltonian $\hat{H}=\hat{H}_{\text {int }}+\hat{H}_{\text {mix }}$, can be used to realize a quantum lock-in amplifier for extraction of the signal $S(t)$. 
Hamiltonian terms if the total Hamiltonian does not commute with itself at different times. Thus, the mix-down process can be achieved by noncommutative operations, and quantum lock-in amplifiers can be achieved.

To illustrate our protocol, we consider an ensemble of $N$ identical two-state bosonic particles. The two states can be selected as any desired two levels, and hereafter we label them as spins $|\uparrow\rangle$ and $|\downarrow\rangle$, respectively. The system states can be well characterized by the collective spin operators: $\hat{J}_{x}=\frac{1}{2}\left(\hat{a}^{\dagger} \hat{b}+\hat{a} \hat{b}^{\dagger}\right), \hat{J}_{y}=\frac{1}{2 i}\left(\hat{a}^{\dagger} \hat{b}-\right.$ $\left.\hat{a} \hat{b}^{\dagger}\right)$, and $\hat{J}_{z}=(1 / 2)\left(\hat{a}^{\dagger} \hat{a}-\hat{b}^{\dagger} \hat{b}\right)$, where $\hat{a}$ and $\hat{b}$ denote annihilation operators for spins $|\uparrow\rangle$ and $|\downarrow\rangle$, respectively. The system states can be represented in terms of the Dicke basis $\{|J, m\rangle\}$, where $\hat{J}_{z}|J, m\rangle=m|J, m\rangle$, with $J=$ $N / 2$ and $m=-J,-J+1, \ldots, J-1, J$. Given the probe as an ensemble of two-mode bosonic particles, the coupling between the probe and the target signal [an ac magnetic field $\mathbf{B}(t)=B \sin (\omega t+\beta) \hat{\mathbf{z}}]$ is described by the Hamiltonian

$$
\hat{H}_{B}(t)=\gamma \mathbf{B}(t) \cdot \mathbf{J}=\gamma B \sin (\omega t+\beta) \hat{J}_{z},
$$

where $B$ corresponds to the magnetic field amplitude, $\gamma$ is the gyromagnetic ratio, $\omega$ denotes the oscillation frequency, and $\beta$ is the initial phase.

Similarly to other lock-in amplifiers, the target signal and the noise couple to the probe through the same physical channel. In the presence of stochastic noise, the interaction between the system and the external ac field is described by the Hamiltonian $\hat{H}_{\text {int }}(t)=M(t) \hat{J}_{z}$, where the external field $M(t)=S(t)+N_{o}(t)$ consists of the target signal $S(t)=$ $S_{0} \sin (\omega t+\beta)$ and the stochastic noise $N_{o}(t)$. The target signal is modulated with time, $\omega$ corresponds to the oscillation frequency, $\beta$ is the initial phase of the signal, and $S_{0}=\gamma B$ stands for the strength of the signal.

To implement the quantum lock-in amplifier, we mix the system with an induced modulation signal that does not commute with $\hat{H}_{\text {int }}$ (see Appendix A for more details). Here we consider the mixing term $\hat{H}_{\text {mix }}(t)=\Omega(t) \hat{J}_{x}$, and the whole Hamiltonian becomes

$$
\hat{H}=\hat{H}_{\text {int }}(t)+\hat{H}_{\text {mix }}(t)=M(t) \hat{J}_{z}+\Omega(t) \hat{J}_{x} .
$$

The noncommutativity of the two modulation terms $\hat{H}_{\text {int }}$ and $\hat{H}_{\text {mix }}$ plays an important role for lock-in measurement. In the unit of $\hbar=1$, the time evolution of system state $|\Psi(t)\rangle$ obeys the Schrödinger equation:

$$
i \frac{\partial|\Psi(t)\rangle}{\partial t}=\left[M(t) \hat{J}_{z}+\Omega(t) \hat{J}_{x}\right]|\Psi(t)\rangle .
$$

In the interaction picture with respect to $\hat{H}_{\text {mix }}$ (see Appendix B for the derivation), the time evolution is described by

$$
\begin{aligned}
i \frac{\partial|\Psi(t)\rangle_{I}}{\partial t} & =\hat{H}_{I}(t)|\Psi(t)\rangle_{I} \\
& =M(t) \cos (\alpha) \hat{J}_{z}+\sin (\alpha) \hat{J}_{y}|\Psi(t)\rangle_{I}
\end{aligned}
$$

where $|\Psi(t)\rangle_{I}=e^{i \int_{0}^{t} \hat{H}_{\text {mix }}\left(t^{\prime}\right) d t^{\prime}}|\Psi(t)\rangle$ and $\alpha=\int_{0}^{t} \Omega\left(t^{\prime}\right) d t^{\prime}$. At time $T$, the system state is

$$
\begin{aligned}
|\Psi(T)\rangle_{I} & =\hat{\mathcal{T}} e^{-i \int_{0}^{T} M(t) \cos (\alpha) \hat{J}_{z}+\sin (\alpha) \hat{J}_{y} d t}|\Psi(0)\rangle_{I} \\
& =\hat{\mathcal{T}} e^{-i\left(\varphi_{1} \hat{J}_{z}+\varphi_{2} \hat{J}_{y}\right)}|\Psi(0)\rangle_{I},
\end{aligned}
$$

with the time-ordering operator $\hat{\mathcal{T}}$, the initial state $|\Psi(0)\rangle_{I}=|\Psi(0)\rangle$, and the two phase factors

$$
\varphi_{1}=S_{0} \int_{0}^{T} \sin (\omega t+\beta) \cos \alpha d t+\int_{0}^{T} N_{o}(t) \cos \alpha d t
$$

and

$$
\varphi_{2}=S_{0} \int_{0}^{T} \sin (\omega t+\beta) \sin \alpha d t+\int_{0}^{T} N_{o}(t) \sin \alpha d t .
$$

If we apply a suitable modulation $\Omega(t)$ to make $\cos \alpha$ and $\sin \alpha$ periodic and synchronized with the signal $S(t)$, the phase accumulated owing to $S(t)$ adds up coherently, whereas the phase accumulated owing to stochastic noise $N_{o}(t)$ can be averaged away. Especially, if the frequencies of the noise spectral components are far from the signal frequency $\omega$, the noise spectral components can be removed in the long-time integration. In this way, the signal-to-noise ratio of the output can be significantly increased. DD is an effective modulation method for quantum control. In the following, we consider the mixing modulation $\Omega(t)$ by DD techniques and demonstrate how to use many-body entanglement to increase the measurement precision.

\section{A. Mixing modulation}

For convenience, we assume $\beta=0$. Our goal is to determine the frequency $\omega$ and the amplitude $B$ via a many-body quantum lock-in amplifier. We first ignore the stochastic noise $N_{o}(t)$; its influences are discussed at the end.

In our scheme, the time-dependent modulation $\Omega(t)$ is designed as a sequence of $\pi$ pulses with equidistant spacing. This technique has been widely used in a singleparticle lock-in amplifier for measuring an oscillating signal in the presence of noise [20,21,23-25]. Generally, a single $\pi$ pulse can be expressed by a rectangular waveform, i.e., $\Omega_{\text {single }}(t)=\frac{\pi}{a}$ for $t_{0} \leq t \leq t_{0}+a$, with $a$ the width (duration) of the single $\pi$ pulse. Ideally, the $\pi$ pulse is assumed to be sharp enough so that $a \rightarrow 0$ and $\Omega_{\text {single }}(t)=\pi \delta\left(t-t_{0}\right)$, where $\delta(t)$ is the Dirac $\delta$ function. For simplicity, we approximate $\Omega(t)$ as a sequence 


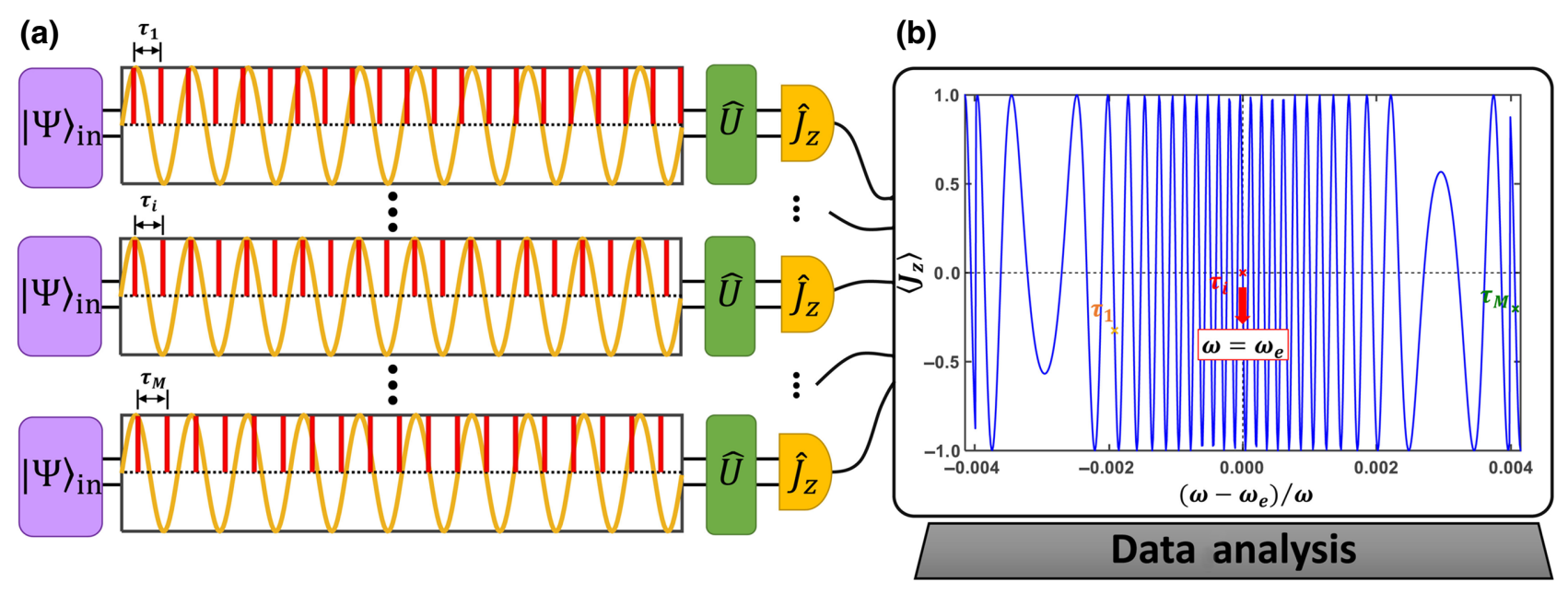

FIG. 2. The general protocol for a many-body quantum lock-in amplifier for detecting an ac magnetic field. (a) The many-body quantum interferometry consists of three stages: (i) preparation, (ii) signal interrogation, and (iii) readout. In the initialization stage, an input state $|\Psi\rangle_{\text {in }}$ is prepared. Then the input state undergoes an interrogation stage for signal accumulation. At this stage, the system state interacts with the magnetic field, and a train of $\pi$ pulses with specific equidistant spacing $\tau_{i}$ is applied. In the readout stage, a certain unitary operation $\hat{U}$ is applied for recombination, and the half-population difference is measured. Here the pulse spacing $\tau_{i}(i=1,2, \ldots, M)$ is adjusted to find the frequency locking point $\omega=\omega_{e}$. (b) The half-population difference versus the detuning $\omega-\omega_{e}$ can give the frequency locking point $\omega=\omega_{e}$. Here, for illustration, we choose a GHZ state with $N=2, T=4 \pi, \omega=20 \pi$, and $\gamma B=2 \pi$.

of equidistant sharp $\pi$ pulses so that it can be written as follows:

$$
\begin{aligned}
\Omega(t) & =\sum_{m=1}^{L} \Omega_{\text {single }}\left[t-\left(m+\frac{1+k}{2}\right) \tau\right] \\
& =\pi \sum_{m=1}^{L} \delta\left[t-\left(m+\frac{1+k}{2}\right) \tau\right], \quad k \in[-1,1),
\end{aligned}
$$

where $\tau$ is the spacing of the adjacent $\pi$ pulses and $L$ denotes the number of pulses. We define the carrier frequency $\omega_{e} \equiv \pi / \tau$, which is analogous to the carrier frequency for the classical lock-in amplifier. $k$ determines the relative phase with respect to the target signal. In the following, we consider $k=0$, which is assumed to be the lock-in condition. When $\omega_{e}=\omega$, the $\pi$ pulses are applied at every peak and valley of the oscillating signal, resulting in a tiny accumulated phase [i.e., Eq. (12)], which can be used for frequency and phase locking. Here, $k$ can be easily tuned by adjusting the start point of the initial pulse. The other condition, $k=-1$, is discussed in Appendix $\mathrm{J}$.

By adjusting the carrier frequency $\omega_{e}$, we can extract the frequency $\omega$ of the ac magnetic field according to the population measurement. For every fixed carrier frequency $\omega_{e}$, one can implement many-body quantum interferometry for measurement, which includes three stages: (i) probe preparation, (ii) signal interrogation, and (iii) readout (see Fig. 2). In the initialization stage, an input state $|\Psi\rangle_{\text {in }}$ is prepared. Then the input state undergoes an interrogation stage for signal accumulation. At this stage, the system state interacts with the ac magnetic field, and a train of $\pi$ pulses with equidistant spacing is applied at the same time. In the readout stage, a certain unitary operation $\hat{U}$ is applied for recombination, and the half-population difference is measured.

To adjust the carrier frequency $\omega_{e}$, one can choose $M$ different pulse spacings $\tau_{i}(i=1,2, \ldots, M)$. The pattern of the half-population difference measurement at time $t=T$ versus the detuning $\omega-\omega_{e}$ will tell us the frequency locking point $\omega=\omega_{e}$ (the red cross in Fig. 2). The pattern depends on the input states (the details for different input states are discussed in Sec. III). Similarly to a classical amplifier, one can also use the time integral of the half-population difference measurement to determine the frequency locking point; of course, this would require many more data. In the following, we consider both the signal for a single interrogation time and the time-averaged signal for multiple interrogation times.

We now discuss the signal interrogation stage. When the evolution time $T$ is fixed, for the $i$ th pulse spacing, the number of $\pi$ pulses is $L=\left[T / \tau_{i}+1 / 2\right]$, where [.] stands for the greatest integer. Substituting Eq. (8) into Eqs. (4) and (5), we obtain the Hamiltonian (1) in the interaction picture:

$$
\hat{H}_{I}=h(t)(\gamma B \sin \omega t) \hat{J}_{z}
$$


where $h(t)$ is the square wave function,

$$
h(t)=\left\{\begin{array}{rlll}
1 & \omega_{e} t+\pi / 2 & \bmod 2 \pi \in[0, \pi), \\
-1 & \omega_{e} t+\pi / 2 & \bmod 2 \pi \in[\pi, 2 \pi) .
\end{array}\right.
$$

In experiments, one can tune $\omega_{e}$ very close to $\omega$ (i.e., $\left.\left|\omega-\omega_{e}\right| \ll \omega\right)$. Under the conditions of $\left|\omega-\omega_{e}\right| \ll \omega$ and $T \gg 2 \pi / \omega$, the Hamiltonian (9) can be approximated by an effective Hamiltonian [23,25]:

$$
\hat{H}_{I}^{\text {eff }}(t)=\frac{2 \gamma B}{\pi} \sin \left[\left(\omega-\omega_{e}\right) t\right] \hat{J}_{z} .
$$

For example, for $T=2 \pi$, the approximation is valid for $\left|\omega-\omega_{e}\right| / \omega<0.1$ for $\omega \geq 10 \pi$; see Appendix C. The output state after the interrogation stage (of duration $T)$ is given as $|\Psi\rangle_{\text {out }}^{I}=e^{-i \int_{0}^{T} \hat{H}_{I}^{\text {eff }}(t) d t}|\Psi\rangle_{\text {in }}=$ $e^{i \int_{0}^{T} \hat{H}_{\text {mix }}(t) d t}|\Psi\rangle_{\text {out }}=e^{i \alpha \hat{J}_{x}}|\Psi\rangle_{\text {out }}$, where $|\Psi\rangle_{\text {out }}$ is the output state in the Schrödinger picture. The two pictures are connected via a unitary transformation $U_{\operatorname{Tr}}=e^{i \alpha \hat{J}_{x}}=e^{i L \pi \hat{J}_{x}}$. The unitary transformation can be easily accomplished via combining multiple $\pi$ pulses, which is a mature technology in quantum control [7-26]; see Appendix E for more details. Moreover, to obtain the analytic calculations for intuitively describing our protocol, we change our description from the Schrödinger picture to the interaction picture. The numerical and analytic results are both very consistent in the two pictures (see Appendix D for more details).

\section{B. Readout}

Finally, in the readout stage, a suitable unitary operation $\hat{U}$ is performed for recombination. The selection of the unitary operation $\hat{U}$ depends on the input state and will influence the measurement precisions, which is discussed in the next section. The final state (in the Schrödinger picture) before the half-population difference measurement can be written as

$$
\begin{aligned}
|\Psi\rangle_{\text {final }} & =\hat{U}|\Psi\rangle_{\text {out }}=\hat{U} e^{-i \alpha \hat{J}_{x}}|\Psi\rangle_{\text {out }}^{I} \\
& =\hat{U} e^{-i \alpha \hat{J}_{x}} e^{-i \int_{0}^{T} \hat{H}_{I}^{\mathrm{eff}}(t) d t}|\Psi\rangle_{\text {in }} .
\end{aligned}
$$

Substituting Eq. (10) into Eq. (11), we have $|\Psi\rangle_{\text {final }}=$ $\hat{U} e^{-i \alpha \hat{J}_{x}} e^{-i \phi \hat{J}_{z}}|\Psi\rangle_{\text {in }}$. Here, the accumulated phase reads

$$
\phi=\frac{2 \gamma B}{\pi} \frac{1-\cos \left[\left(\omega-\omega_{e}\right) T\right]}{\omega-\omega_{e}} .
$$

The expectation of the half-population difference measurement on the final state is

$$
\begin{aligned}
\left\langle\hat{J}_{z}\right\rangle_{f} & =\left\langle\left.\Psi\right|_{\text {final }} \hat{J}_{z} \mid \Psi\right\rangle_{\text {final }}=\left\langle\left.\Psi\right|_{\text {out }} ^{I} \hat{U}_{I}^{\dagger} \hat{J}_{z}^{I} \hat{U}_{I} \mid \Psi\right\rangle_{\text {out }}^{I} \\
& =\left\langle\left.\Psi\right|_{\text {final }} ^{I} \hat{J}_{z}^{I} \mid \Psi\right\rangle_{\text {final }}^{I},
\end{aligned}
$$

with $\hat{J}_{z}^{I}=e^{i \alpha \hat{J}_{x}} \hat{J}_{z} e^{-i \alpha \hat{J}_{x}}$ and $\hat{U}_{I}=e^{i \alpha \hat{J}_{x}} \hat{U}^{-i \alpha \hat{J}_{x}}$. At time $T$, we have $\alpha=L \pi$ and $\hat{U}_{I}=e^{i L \pi \hat{J}_{x}} \hat{U} e^{-i L \pi \hat{J}_{x}}$. When the pulse number $L$ is even, $\hat{J}_{x}^{I}=\hat{J}_{x}, \hat{J}_{y}^{I}=\hat{J}_{y}$, and $\hat{J}_{z}^{I}=\hat{J}_{z}$; when $L$ is odd, $\hat{J}_{x}^{I}=\hat{J}_{x}, \hat{J}_{y}^{I}=-\hat{J}_{y}$, and $\hat{J}_{z}^{I}=-\hat{J}_{z}$ (see Appendix E for more details). Thus, according to Eq. (13), one can easily obtain the half-population difference measurement on the final state via $|\Psi\rangle_{\text {out }}^{I}$. When the pulse number $L$ is even, $\left\langle\hat{J}_{z}\right\rangle_{f}=\left\langle\left.\Psi\right|_{\text {out }} ^{I} \hat{J}_{z} \mid \Psi\right\rangle_{\text {out }}^{I}$. When $L$ is odd, $\left\langle\hat{J}_{z}\right\rangle_{f}=$ $-\left\langle\left.\Psi\right|_{\text {out }} ^{I} \hat{J}_{z} \mid \Psi\right\rangle_{\text {out }}^{I}$. The odd-even nature of the pulse number influences the expectation of the half-population difference. This will cause a jump at the location whenever the corresponding pulse number changes. Furthermore, to eliminate the influence of the odd-even nature of $L$ on the half-population difference measurement, we define a measurement signal $J_{z}$ as

$$
J_{z}=(-1)^{K}\left\langle\hat{J}_{z}\right\rangle_{f},
$$

with $K=0$ for even $L$ and $K=1$ for odd $L$. The measurement signal $J_{z}$ is dependent on the half-population difference measurement and the pulse number $L$, and thus it does not add any complexity to existing measurement techniques. Meanwhile, the square of the half-population difference on the final state can also be explicitly written via $|\Psi\rangle_{\text {out }}^{I}$, and it is $\left\langle\hat{J}_{z}^{2}\right\rangle_{f}=\left\langle\left.\Psi\right|_{\text {out }} ^{I} \hat{J}_{z}^{2} \mid \Psi\right\rangle_{\text {out }}^{I}$. We now illustrate the basis of the lock-in amplifier in detail. According to Eq. (12), when $\omega-\omega_{e} \rightarrow 0$, the accumulated phase $\phi \rightarrow \gamma B\left(\omega-\omega_{e}\right) T^{2} / \pi \approx 0$ and the value of $J_{z}$ is time independent, while $\hat{J}_{z}$ is time dependent for $\omega-\omega_{e} \neq 0$. This property can be used to find the frequency locking point $\omega=\omega_{e}$. For the property of amplification, we consider the Hamiltonian for the system coupled to the external ac magnetic field in noise and described as

$$
\hat{H}_{B}=\left[\gamma B \sin \omega t+N_{o}(t)\right] \hat{J}_{z},
$$

where $N_{o}(t)$ is the stochastic noise. Because the modulation term $\hat{H}_{\text {mix }}=\Omega(t) \hat{J}_{x}$ is a set of sharp $\pi$ pulses, we have $\alpha=\int_{0}^{t} \Omega\left(t^{\prime}\right) d t^{\prime}=L \pi$, so $\cos \alpha= \pm 1$ and $\sin \alpha=0$. From Eqs. (6) and (7), we have

$$
\begin{aligned}
\varphi_{1} & =\int_{0}^{T}\left[\gamma B \sin \omega t+N_{o}(t)\right] \cos \alpha d t \\
& =\int_{0}^{T} \gamma B \sin \omega t \cos \alpha d t+\int_{0}^{T} N_{o}(t) \cos \alpha d t \\
\varphi_{2} & =0
\end{aligned}
$$

Thus, $\int_{0}^{T} N_{o}(t) \cos \alpha d t \approx 0, \varphi_{1} \approx \phi$. The effects of the stochastic noise $N_{o}(t)$ are canceled out through the time integral. Meanwhile, the contribution of the target signal $S_{0} \sin \omega t$ is imprinted on the phase $\phi$, which is sensitively dependent on the detuning $\omega-\omega_{e}$. The signal-to-noise ratio can be effectively increased with our many-body quantum amplifier. 


\section{MEASUREMENT PRECISIONS}

In this section, we analyze the measurement precisions of our many-body quantum lock-in amplifier and show how entanglement can increase the measurement precisions. Within our scheme, for individual particles without entanglement, the measurement precisions can approach the SQL. For entangled particles in a spin cat state, the measurement precisions can be increased to the HL. We also discuss the robustness of our many-body quantum lock-in amplifier against stochastic noise.

According to the parameter quantum estimation theory, the precision of the parameter is constrained by the quantum Cramér-Rao bound (QCRB) [1,57-59],

$$
\Delta \mu \geq \Delta \mu_{\mathrm{QCRB}} \equiv \frac{1}{\sqrt{\eta F_{Q}^{\mu}}} \quad(\mu=\omega, B),
$$

which is characterized by the quantum Fisher information (QFI):

$$
F_{Q}^{\mu}=4\left(\left\langle\partial_{\mu} \Psi_{\text {out }} \mid \partial_{\mu} \Psi_{\text {out }}\right\rangle-\left|\left\langle\partial_{\mu} \Psi_{\text {out }} \mid \Psi\right\rangle_{\text {out }}\right|^{2}\right),
$$

where $\eta$ corresponds to the number of trials and $\left|\partial_{\mu} \Psi_{\text {out }}\right\rangle$ denotes the partial derivative of $|\Psi\rangle_{\text {out }}$ with respect to the parameter $\mu$. Within our scheme, the expression for the QFI can be simplified to

$$
\begin{aligned}
F_{Q}^{\mu} & =4\left(\frac{\partial \phi}{\partial \mu}\right)^{2}\left[{ }_{\text {in }}\left\langle\Psi\left|\hat{J}_{z}^{2}\right| \Psi\right\rangle_{\text {in }}-\left({ }_{\text {in }}\left\langle\Psi\left|\hat{J}_{z}\right| \Psi\right\rangle_{\text {in }}\right)^{2}\right] \\
& =4\left(\frac{\partial \phi}{\partial \mu}\right)^{2}\left(\Delta \hat{J}_{z}\right)_{\text {in }}^{2} .
\end{aligned}
$$

Thus, the properties of initial states determine the ultimate measurement precisions. As shown below, for individual particles, $\left(\Delta \hat{J}_{z}\right)_{\text {in }}^{2} \propto N$, while for entangled particles, $\left(\Delta \hat{J}_{z}\right)_{\text {in }}^{2} \propto N^{2}$, showing the entanglement has the ability to increase the measurement precision from the SQL to the Heisenberg limit.

To further characterize the measurement precision of $\omega$ and $B$, we use the error propagation formula [1,57-59]. The measurement precisions for $\omega$ and $B$ are

$$
\Delta \omega=\frac{\left(\Delta \hat{J}_{z}\right)_{f}}{\left|\partial\left\langle\hat{J}_{z}\right\rangle_{f} / \partial \omega_{e}\right|}
$$

and

$$
\Delta B=\frac{\left(\Delta J_{z}\right)_{f}}{\left|\partial\left\langle\hat{J}_{z}\right\rangle_{f} / \partial B\right|}
$$

where

$$
\left(\Delta \hat{J}_{z}\right)_{f}=\sqrt{\left\langle\hat{J}_{z}^{2}\right\rangle_{f}-\left\langle\hat{J}_{z}\right\rangle_{f}^{2}} .
$$

\section{A. Individual particles}

We first consider individual particles without any entanglement. Suppose all the particles are prepared in the spin coherent state (SCS) $|\Psi\rangle_{\mathrm{SCS}}=e^{-i(\pi / 2) \hat{J}_{y}}|N / 2,-N / 2\rangle$. This input state can be easily generated by applying a $\pi / 2$ pulse on the state of all particles in the spin-down state $|\downarrow\rangle$. In this situation, one can choose $\hat{U}_{I}=e^{-i(\pi / 2) \hat{J}_{y}}$. Then the final state before the half-population difference measurement can be written as

$$
|\Psi\rangle_{\text {final }}^{I}=e^{-i \frac{\pi}{2} \hat{J}_{y}} e^{-i \phi \hat{J}_{z}}|\Psi\rangle_{\mathrm{SCS}} .
$$

In an explicit form, the final state becomes

$$
|\Psi\rangle_{\text {final }}^{I}=\sum_{m=-J}^{J} \sqrt{C_{J}^{m}}\left(\cos \frac{\phi}{2}\right)^{J+m}\left(i \sin \frac{\phi}{2}\right)^{J-m}|J, m\rangle,
$$

where $C_{J}^{m}=[(2 J) ! /(J+m) !(J-m) !]$ is the binomial coefficient. According to Eq. (19), the QFI for the two parameter with SCS can be written as

$$
F_{Q}^{\omega}=\left[\frac{2 \gamma B}{\pi} \frac{G}{\left(\omega-\omega_{e}\right)^{2}}\right]^{2} N
$$

and

$$
F_{Q}^{B}=N\left\{\frac{2 \gamma}{\pi} \frac{1-\cos \left[\left(\omega-\omega_{e}\right) T\right]}{\omega-\omega_{e}}\right\}^{2},
$$

with

$$
G=\cos \left[\left(\omega-\omega_{e}\right) T\right]+T\left(\omega-\omega_{e}\right) \sin \left[\left(\omega-\omega_{e}\right) T\right]-1 .
$$

Obviously, for both parameters $\omega$ and $B$, the ultimate precision bounds with individual particles can attain the SQL (i.e., $\Delta \omega_{\mathrm{QCRB}} \propto 1 / \sqrt{N}$ and $\Delta B_{\mathrm{QCRB}} \propto 1 / \sqrt{N}$ ). Furthermore, we consider the measurement precision via halfpopulation difference measurement. After some algebra, the expectations of the half-population difference and the square of the half-population difference on the final state can be explicitly written as $\left\langle\hat{J}_{z}\right\rangle_{f}=(-1)^{L}(N / 2) \cos \phi$ and $\left\langle\hat{J}_{z}^{2}\right\rangle_{f}=(N / 4)+[N(N-1) / 4] \cos ^{2} \phi$. Thus, according to Eq. (14), the measurement signal $J_{z}$ is given as

$$
J_{z}=\frac{N}{2} \cos \left\{\frac{2 \gamma B}{\pi} \frac{1-\cos \left[\left(\omega-\omega_{e}\right) T\right]}{\omega-\omega_{e}}\right\} .
$$

The information for the estimated parameters $\omega$ and $B$ can be inferred from the pattern of the measurement signal $J_{z}$. As Eq. (28) is exactly symmetric with respect to the lock-in point $\omega-\omega_{e}=0$, one can determine the lock-in 
point from the symmetry of the measurement signal $J_{z}$ with respect to the lock-in point; see Figs. 3(a) and 3(d). The analytic expression is consistent with the numerical result (see Appendix D). In particular, the measurement signals $J_{z}$ for different evolution times $T$ have the same value at the lock-in point, and thus one can also determine the lockin point from this crossover point; see Fig. 3(a). Once the value of $\omega$ is determined, one can extract $B$ from Eq. (28) via a fitting procedure. Therefore, the values of $\omega$ and $B$ can be simultaneously obtained by measuring only $J_{z}$ in experiments.

In general, for a large particle number, the pattern of the measurement signal $J_{z}$ becomes a high-frequency oscillation and it is not easy to determine the lock-in point from the pattern symmetry in experiments. To address this, one can determine the lock-in point via the time-averaged signal $\tilde{J}_{z}$ :

$$
\tilde{J}_{z}=\frac{1}{T_{2}-T_{1}} \int_{T_{1}}^{T_{2}} J_{z} d t
$$

Substituting Eq. (28) into Eq. (29), in the vicinity of the lock-in point $\omega-\omega_{e}=0$, one can analytically obtain

$$
\begin{aligned}
\tilde{J}_{z} \approx & \frac{N \pi}{2 \sqrt{2}\left(T_{2}-T_{1}\right)} \sqrt{\frac{1}{\gamma B\left(\omega-\omega_{e}\right)}} \\
& \times\left\{\mathfrak{F}_{C}\left[\sqrt{\frac{\gamma B\left(\omega-\omega_{e}\right)}{2}} T_{2}\right]-\mathfrak{F}_{C}\left[\sqrt{\frac{\gamma B\left(\omega-\omega_{e}\right)}{2}} T_{1}\right]\right\},
\end{aligned}
$$

where $\mathfrak{F}_{C}(\cdot)$ is the Fresnel cosine integral function. For a SCS, Eq. (30) is symmetric with respect to the lockin point for fixed $B$; see Fig. $3(\mathrm{~g})$. The analytic results (dashed red line) are very consistent with the numerical results (solid blue line) in a wide region around the lock-in point. This means that one can determine the values of $\omega$ and $B$ via the time-averaged signal $\tilde{J}_{z}$ in experiments; of course, this would require more experimental data.

One can analytically obtain $\Delta \omega$ and $\Delta B$. They read

$$
\Delta \omega=\frac{\pi}{2 \gamma B \sqrt{N}} \frac{\left(\omega-\omega_{e}\right)^{2}}{|G|}
$$
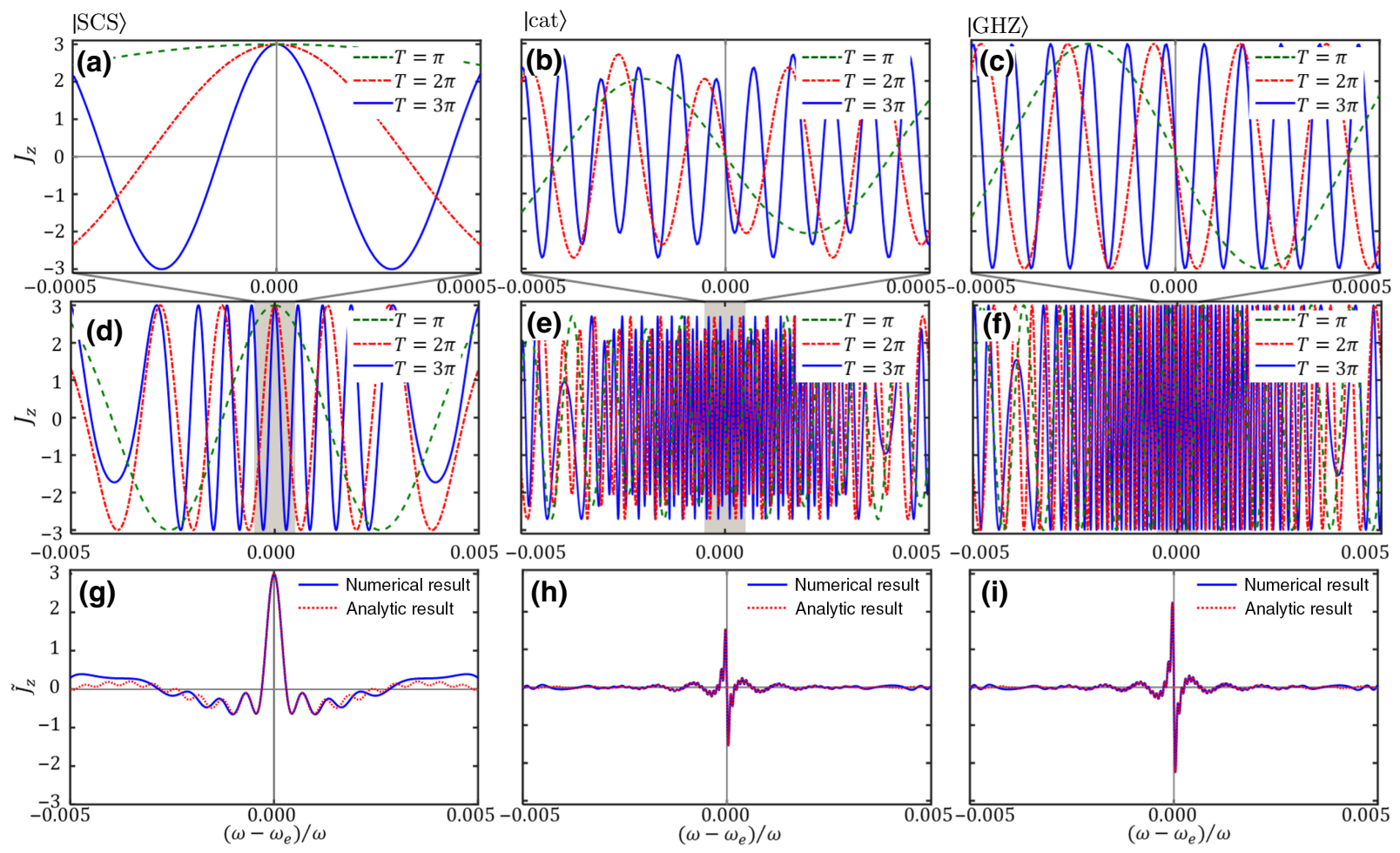

FIG. 3. The lock-in signal of the many-body quantum lock-in amplifier. The measurement signal $J_{z}$ versus the detuning $\omega-\omega_{e}$ for (a),(d) the SCS, (b),(e) the spin cat state $|\Psi(\theta=\pi / 8)\rangle_{\text {cat }}$, and (c),(f) the GHZ state $|\Psi(\theta=0)\rangle_{\text {cat }}$ for different evolution times $T$. The measurement signals $J_{z}$ are symmetric (or antisymmetric) with respect to the lock-in point $\omega-\omega_{e}=0$ and they are both consistent with the analytic expressions [Eqs. (28), (38), and (41)]. The time-averaged signal $\tilde{J}_{z}=\frac{1}{T_{2}-T_{1}} \int_{T_{1}}^{T_{2}} J_{z} d t$ versus the detuning $\omega-\omega_{e}$ for (g) the SCS, (h) the spin cat state $|\Psi(\theta=\pi / 8)\rangle_{\text {cat }}$, and (i) the GHZ state $|\Psi(\theta=0)\rangle_{\text {cat }}$, with $T_{1}=\pi$ and $T_{2}=3 \pi$. The time-averaged signal $\tilde{J}_{z}$ is symmetric (or antisymmetric) with respect to the lock-in point $\omega-\omega_{e}=0$. The analytic results (dashed red line) fit well with the numerical results (solid blue line) in a wide range around the lock-in point. Here $\omega=20 \pi, \gamma B=2 \pi$, and $N=6$. 

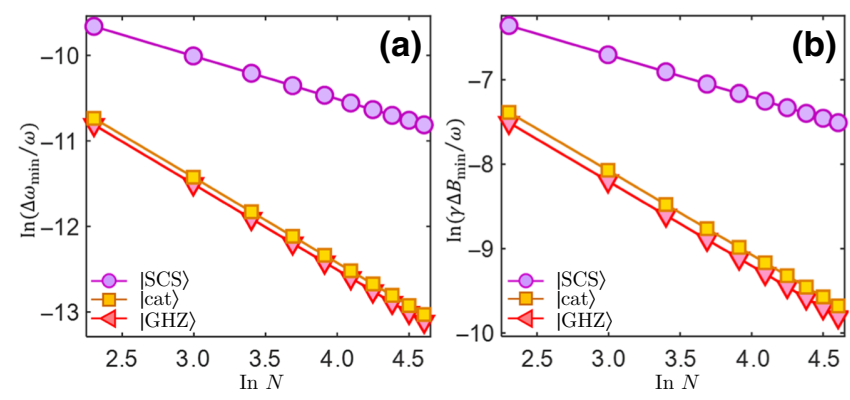

FIG. 4. Log-log scaling of the optimal measurement precisions (a) $\Delta \omega$ and (b) $\Delta B$ versus total particle number. The circles are the results for a SCS with $\hat{U}_{I}=e^{-i \frac{\pi}{2} \hat{J}_{y}}$. The squares and triangles denote the results for the spin cat state $|\Psi(\theta=\pi / 8)\rangle_{\text {cat }}$ and the GHZ state $|\Psi(\theta=0)\rangle_{\text {cat }}$, respectively, with $\hat{U}_{I}=e^{i \frac{\pi}{2} \hat{J}_{x}} e^{i \frac{\pi}{2} \hat{J}_{z}^{2}} e^{i \frac{\pi}{2} \hat{J}_{x}}$. The lines are the corresponding fitting curves. Here $T=2 \pi, \omega=$ $20 \pi,\left|\left(\omega-\omega_{e}\right) / \omega\right| \in[0,0.008]$, and $\gamma B \in[0,2 \pi]$.

and

$$
\Delta B=\frac{\pi}{2 \gamma \sqrt{N}}\left|\frac{\omega-\omega_{e}}{\cos \left[\left(\omega-\omega_{e}\right) T\right]-1}\right|,
$$

From Eqs. (31) and (32), the measurement precisions $\Delta \omega$ and $\Delta B$ for individual particles exhibit only SQL scaling. For fixed $N$, the measurement precision $\Delta \omega$ is dependent on the detuning $\omega-\omega_{e}$, the signal amplitude $B$, and the evolution time $T$ (see Appendix $\mathrm{H}$ for more details). When $\omega-\omega_{e} \rightarrow 0, \Delta \omega \rightarrow \pi / 3 \gamma B T^{2} \sqrt{N}$ attains the optimal value. However, the measurement precision $\Delta B$ is dependent only on the detuning $\omega-\omega_{e}$ and $T$. When $\omega-$ $\omega_{e} \rightarrow 0, \Delta B \rightarrow \pi / \gamma\left(\omega-\omega_{e}\right) T^{2} \sqrt{N} \rightarrow \infty$, the measurement precision $\Delta B$ is diverged. When $\cos \left[\left(\omega-\omega_{e}\right) T\right]+$ $\left(\omega-\omega_{e}\right) T \sin \left[\left(\omega-\omega_{e}\right) T\right]=1, \Delta B$ attains its minimum.

We numerically find the optimal measurement precisions in the range $\left|\left(\omega-\omega_{e}\right) / \omega\right| \in[0,0.008]$ and calculate the corresponding scaling versus particle number for $\gamma B=$ $2 \pi$. According to the fitting results (see Fig. 4), the log$\log$ measurement precisions $\ln \left(\Delta \omega_{\min } / \omega\right) \approx-0.5 \ln N-$ 8.51 and $\ln \left(\gamma \Delta B_{\min } / \omega\right) \approx-0.5 \ln N-5.20$. For the two parameters, the optimal measurement precisions $\Delta \omega_{\text {min }}$ [purple circles in Fig. 4(a)] and $\Delta B_{\min }$ [purple circles in Fig. 4(b)] both exhibit SQL scaling as expected.

\section{B. Entangled particles}

In this subsection, we discuss how to realize the Heisenberg-limited measurement for $\omega$ and $B$ within this framework. Entanglement is a useful quantum resource to increase the measurement precision. A spin cat state, as a kind of non-Gaussian entangled state, is a promising candidate for entanglement-enhanced metrology $[32,36$, $51,60-62]$. Here we demonstrate how to use spin cat states to realize an entanglement-enhanced quantum many-body lock-in amplifier.
Spin cat states are typical kinds of macroscopic superposition of spin coherent states (MSSCS) [36,51]. For total particle number $N \geq 6$, when $\theta \leq \pi / 8$ [51], the corresponding MSSCS can be regarded as a spin cat state (see Appendix G). Under this condition, we approximate the spin cat states as

$$
|\Psi(\theta)\rangle_{\mathrm{cat}}=\frac{1}{\sqrt{2}}\left[\sum_{m=-J}^{J} c_{m}^{J}(\theta)(|J, m\rangle+|J,-m\rangle)\right],
$$

where $c_{m}^{J}(\theta)=\sqrt{[(2 J) ! /(J+m) !(J-m) !]} \cos ^{J+m}(\theta / 2)$ $\sin ^{J-m}(\theta / 2)$. Especially, when $\theta=0$, the spin cat state $|\Psi(0)\rangle_{\text {cat }}=(1 / \sqrt{2})(|J, J\rangle+|J,-J\rangle)$ corresponds to the well-known GHZ state. According to Eq. (19), the QFI for the two parameters with spin cat states can be written as

$$
F_{Q}^{\omega}=\left[\frac{2 \gamma B}{\pi C(\theta)} \frac{G}{\left(\omega-\omega_{e}\right)^{2}}\right]^{2} N^{2}
$$

and

$$
F_{Q}^{B}=\left[\frac{2 \gamma}{\pi C(\theta)} \frac{1-\cos \left[\left(\omega-\omega_{e}\right) T\right]}{\omega-\omega_{e}}\right]^{2} N^{2} .
$$

The coefficient $C(\theta)=1+\left[2 \tan ^{2}(\theta / 2) /\left(1+\tan ^{2}(\theta / 2)\right)\right]$ does not depend on the particle number $N$ [51]. By use of entangled particles in spin cat states, the ultimate precision bounds can be increased to the HL (i.e., $\Delta \omega_{\mathrm{QCRB}} \propto 1 / N$ and $\left.\Delta B_{\mathrm{QCRB}} \propto 1 / N\right)$.

Interaction-based readout is a powerful technique for achieving the HL via spin cat states without singleparticle-resolved detection [46-56,63], which is now feasible in experiments [14,55]. Similarly, we try to adopt an interaction-based operation $\hat{U}_{I}=e^{i(\pi / 2) \hat{J}_{x}} e^{i(\pi / 2) \hat{J}_{z}^{2}} e^{i(\pi / 2) \hat{J}_{x}}$ in the readout stage.

Therefore, the final state before measurement of the half-population difference can be written as

$$
|\Psi\rangle_{\text {final }}^{I}=e^{i \frac{\pi}{2} \hat{J}_{x}} e^{i \frac{\pi}{2} \hat{J}_{z}^{2}} e^{i \frac{\pi}{2} \hat{J}_{x}} e^{-i \phi}|\Psi(\theta)\rangle_{\text {cat }} .
$$

When $N$ is an even number, the final state $|\Psi\rangle_{\text {final }}^{I}$ has an analytic form that is written as

$$
\begin{aligned}
|\Psi\rangle_{\text {final }}^{I}= & \frac{\sqrt{2}}{4}(-1)^{J} \sum_{m=-J}^{m=J}\left[c_{m}^{J}(\theta)+c_{-m}^{J}(\theta)\right] \\
& \times\left\{\left[1+(-1)^{m}\right](\cos m \phi+\sin m \phi)\right. \\
& \left.\times-i\left[1-(-1)^{m}\right](\cos m \phi-\sin m \phi)\right\}|J, m\rangle .
\end{aligned}
$$

The expectations of the half-population difference $\left\langle J_{z}\right\rangle_{f}=$ $(-1)^{L+1} \sum_{m=-J}^{m=J}(-1)^{J-m} m\left|c_{m}^{J}(\theta)\right|^{2} \sin 2 m \phi$, and thus we 
have

$$
J_{z}=-\sum_{m=-J}^{m=J}(-1)^{J-m} m\left|c_{m}^{J}(\theta)\right|^{2} \sin 2 m \phi .
$$

Because of the entanglement, the oscillation of the measurement signal $J_{z}$ becomes related to $2 m$. The expectation of $\hat{J}_{z}^{2}$ is $\left\langle\hat{J}_{z}^{2}\right\rangle_{f}=\sum_{m=-J}^{m=J} m^{2}\left|c_{m}^{J}(\theta)\right|^{2}$. Equation (38) is also exactly antisymmetric with respect to the lock-in point $\omega-\omega_{e}=0$; see the example for a spin cat state with $\theta=\pi / 8$ in Figs. 3(b) and 3(e). Obviously, the analytic expression is very consistent with the numerical results (see Appendix D). Correspondingly, one can determine the lock-in point from the symmetry property of the measurement signal $J_{z}$. Similarly, the measurement signals $J_{z}$ for different evolution times $T$ have the same value at the lockin point. Substituting Eq. (38) into Eq. (29), in the vicinity of the lock-in point $\omega-\omega_{e}=0$, we find the corresponding time-averaged signal reads

$$
\begin{aligned}
\tilde{J}_{z} \approx & -\frac{\pi}{2\left(T_{2}-T_{1}\right)} \frac{1}{\sqrt{\gamma B\left(\omega-\omega_{e}\right)}} \\
& \times \sum_{m=-J}^{m=J}(-1)^{J-m} \sqrt{m}\left|c_{m}^{J}(\theta)\right|^{2} \\
& \times\left\{\mathfrak{F}_{S}\left[\sqrt{2 m \gamma B\left(\omega-\omega_{e}\right)} T_{2}\right]\right. \\
& \left.-\mathfrak{F}_{S}\left[\sqrt{2 m \gamma B\left(\omega-\omega_{e}\right)} T_{1}\right]\right\},
\end{aligned}
$$

where $\mathfrak{F}_{S}(\cdot)$ is the Fresnel sine integral function. For a spin cat state, Eq. (39) is antisymmetric with respect to the lockin point for fixed $B$ and the symmetry point is more obvious and easy to extract, as shown in Fig. 3(h). The analytic results (dashed red line) fit well with the numerical ones (solid blue line) in a wide region around the lock-in point. Thus, one can determine the values of $\omega$ and $B$ via the timeaveraged signal $\tilde{J}_{z}$ in experiments.

When $\theta=0$, the spin cat state corresponds to the GHZ state. According to Eq. (37), the final state $|\Psi\rangle_{\text {final }}^{I}$ becomes

$$
\begin{aligned}
|\Psi\rangle_{\text {final }}^{I}= & \frac{1}{\sqrt{2}}(\cos J \phi-\sin J \phi)|J, J\rangle \\
& +\frac{1}{\sqrt{2}}(\cos J \phi+\sin J \phi)|J,-J\rangle .
\end{aligned}
$$

Thus the expectation $\left\langle J_{z}\right\rangle_{f}=-(N / 2)(-1)^{L} \sin N \phi$, and the measurement signal reads

$$
J_{z}=-\frac{N}{2} \sin N \phi .
$$

Clearly, the main frequency of the oscillation of $J_{z}$ becomes proportional to $N=2 J$. Equation (41) is antisymmetric with respect to $\omega-\omega_{e}$, which can be used to locate the zero detuning point $\omega=\omega_{e}$, as shown in Figs. 3(c) and 3(f). The analytic expression is consistent with the numerical result (see Appendix D). The time-averaged signal $\tilde{J}_{z}$ near the lock-in point becomes

$$
\begin{aligned}
\tilde{J}_{z} \approx & -\frac{\pi}{2 \sqrt{2}\left(T_{2}-T_{1}\right)} \sqrt{\frac{N}{\gamma B\left(\omega-\omega_{e}\right)}} \\
& \times\left\{\mathfrak{F}_{S}\left[\sqrt{\frac{N \gamma B\left(\omega-\omega_{e}\right)}{2}} T_{2}\right]\right. \\
& \left.\times-\mathfrak{F}_{S}\left[\sqrt{\frac{N \gamma B\left(\omega-\omega_{e}\right)}{2}} T_{1}\right]\right\} .
\end{aligned}
$$

It is obvious that Eq. (42) is antisymmetric with respect to the lock-in point for fixed $B$ and the symmetry point located at $\omega-\omega_{e}=0$, as shown in Fig. 3(i). Similarly, the analytic results (dashed red line) fit well with the numerical results (solid blue line) in a wide region around the lock-in point. Thus, one can simultaneously determine the values of $\omega$ and $B$ via the time-averaged signal $\tilde{J}_{z}$ in experiments. Moreover, the square of the half-population difference is independent of the two parameters, i.e., $\left\langle J_{z}^{2}\right\rangle_{f}=J^{2}=$ $N^{2} / 4$. According to Eqs. (20) and (21), the measurement precisions for $\omega$ and $B$ can be analytically obtained:

$$
\begin{aligned}
& \Delta \omega=\frac{\pi}{2 \gamma B N} \frac{\left(\omega-\omega_{e}\right)^{2}}{|G|}, \\
& \Delta B=\frac{\pi}{2 \gamma N}\left|\frac{\left(\omega-\omega_{e}\right)}{\cos \left[\left(\omega-\omega_{e}\right) T\right]-1}\right| .
\end{aligned}
$$

Similarly to the case of a SCS, for fixed $N$, the measurement precision $\Delta \omega$ is dependent on the detuning $\omega-\omega_{e}$, the signal amplitude $B$, and the evolution time $T$ (see Appendix $\mathrm{H}$ for more details). When $\omega-\omega_{e} \rightarrow 0, \Delta \omega \rightarrow$ $\pi / 3 \gamma B T^{2} N$ attains the optimal value, which achieves the HL. The measurement precision $\Delta B$ is dependent only on the detuning $\omega-\omega_{e}$ and the evolution time $T$. When $\omega-\omega_{e} \rightarrow 0, \Delta B \rightarrow \pi / \gamma\left(\omega-\omega_{e}\right) T^{2} N \rightarrow \infty$, the measurement precision $\Delta B$ is diverged. Also, when $\cos [(\omega-$ $\left.\left.\omega_{e}\right) T\right]+\left(\omega-\omega_{e}\right) T \sin \left[\left(\omega-\omega_{e}\right) T\right]=1, \Delta B$ attains its optimal value. For other spin cat states, one can analytically obtain the measurement precisions $\Delta \omega$ and $\Delta B$ (see Appendix $\mathrm{H}$ for more details). They are written as

$$
\begin{aligned}
\Delta \omega & =\frac{\tilde{A} \pi}{2 \gamma B} \frac{\left(\omega-\omega_{e}\right)^{2}}{|G|}, \\
\Delta B & =\frac{\tilde{A} \pi}{2 \gamma}\left|\frac{\omega-\omega_{e}}{\cos \left[\left(\omega-\omega_{e}\right) T\right]-1}\right|,
\end{aligned}
$$

where 


$$
\tilde{A}=\frac{\sqrt{\sum_{m=-J}^{m=J} m^{2}\left|c_{m}^{J}(\theta)\right|^{2}-\left[\sum_{m=-J}^{m=J}(-1)^{J-m} m\left|c_{m}^{J}(\theta)\right|^{2} \sin 2 m \phi\right]^{2}}}{\left.\left|2 \sum_{m=-J}^{m=J} m^{2}(-1)^{J-m}\right| c_{m}^{J}(\theta)\right|^{2} \cos 2 m \phi \mid} .
$$

In particular, when $\phi=(2 \gamma B / \pi)\left\{\left(1-\cos \left[\left(\omega-\omega_{e}\right) T\right]\right) /\right.$ $\left.\left(\omega-\omega_{e}\right)\right\}=\pi / 2, \sin m \pi=0$, and $(-1)^{J-m} \cos m \pi=1$, the measurement precisions $\Delta \omega$ and $\Delta B$ can be simplified as $\left.\Delta \omega\right|_{\phi=\pi / 2}=C(\theta)(\pi / 2 \gamma B N)\left[\left(\omega-\omega_{e}\right)^{2} /|G|\right]$ and $\left.\Delta B\right|_{\phi=\pi / 2}=C(\theta)(\pi / 2 \gamma N) \mid\left\{\left(\omega-\omega_{e}\right) /\left(\cos \left[\left(\omega-\omega_{e}\right) T\right]\right.\right.$ $-1)\} \mid$. The case of $\omega-\omega_{e} \rightarrow 0$ is discussed in Appendix I.

Furthermore, we numerically find the optimal measurement precisions in the range $\left|\left(\omega-\omega_{e}\right) / \omega\right| \in[0,0.008]$ and calculate the corresponding scaling versus particle number for the spin cat state $|\Psi(\theta=\pi / 8)\rangle_{\text {cat }}$ and the GHZ state. As shown in Fig. 4, for the spin cat state $|\Psi(\theta=\pi / 8)\rangle_{\text {cat }}$, the optimal log-log measurement precisions $\Delta \omega_{\min }$ [yellow squares in Fig. 4(a)] and $\Delta B_{\min }$ [yellow squares in Fig. 4(b)] are $\ln \left(\Delta \omega_{\min } / \omega\right) \approx$ $-\ln N-8.44$ and $\ln \left(\gamma \Delta B_{\min } / \omega\right) \approx-\ln N-5.09$. For the GHZ state, the optimal log-log measurement precisions $\Delta \omega_{\min }$ [red triangles in Fig. $\left.4(\mathrm{a})\right]$ and $\Delta B_{\min }[\mathrm{red}$ triangles in Fig. $4(\mathrm{~b})]$ are $\ln \left(\Delta \omega_{\min } / \omega\right) \approx-\ln N-8.51$ and $\ln \left(\gamma \Delta B_{\min } / \omega\right) \approx-\ln N-5.20$. It is indicated that the measurement precisions $\Delta \omega$ and $\Delta B$ for spin cat states can exhibit Heisenberg-limited scaling.

\section{Robustness against stochastic noise}

Below we verify the robustness of our many-body quantum lock-in amplifier against stochastic noise by numerical simulations. The signal noise, which is made up of extraneous information, will cause the target signal to fluctuate and hence affect the signal extraction. It commonly exists in precision measurement experiments [64-68]. Under the influence of signal noise, the total signal $M(t)$ becomes

$$
M(t)=S(t)+N_{o}(t),
$$

with the target signal $S(t)=S_{0} \sin \omega t$ and the noise $N_{o}(t)$. Given that the signal and the noise couple to the probe through the same physical channel, if the initial state is a pure state, one still can describe the time evolution via the Schrödinger equation by substituting Eq. (48) into Eq. (3).

One of the most common and most dominant types of signal noise is white noise [69]. To illustrate the robustness of our scheme, we assume $N_{o}(t)$ is white noise satisfying $N_{o}(t) \in[-\eta, \eta]$, where $\eta$ is the maximum fluctuation strength. For such white noise, its long-time integration $\overline{N_{o}(t)}=0$. The signal without noise and the noisy signals with $\eta=5 \gamma B$ and $\eta=25 \gamma B$ are shown in Appendix F.
According to Eq. (16), the effect of white noise can be canceled out. To verify the analytic results, we illustrate the robustness of our scheme directly. We numerically calculate the measurement precision of $\Delta \omega$ versus detuning under different stochastic noise $N_{o}(t)$ with $\eta=0, \eta=$ $5 \gamma B, \sim \eta=25 \gamma B$; see Figs. 5(a) and 5(c). We choose a small range in the vicinity of zero detuning and after averaging 20 times. For the SCS and the GHZ state, with modest noise strength $\eta=5 \gamma B, \Delta \omega$ will blow up near the locations where $d\left\langle J_{z}\right\rangle_{f} / d \omega_{e}=0$. However, the stochastic noise hardly affects the measurement precisions at other locations. For the spin cat state, with modest noise strength $\eta=5 \gamma B$, the curves are almost identical to the ones for the corresponding ideal cases. When the noise strength is extremely strong $\eta=25 \gamma B$, only small fluctuations appear and the negative impact of the noise can still be mostly eliminated. Our numerical simulation clearly indicates that even though extreme stochastic noise exist, our quantum many-body lock-in amplifier can still be used for alternating signal detection with a high signal-to-noise ratio. Moreover, the spin cat states can still be exploited to achieve the Heisenberg-limited quantum lock-in amplifier, which is feasible in realistic experiments.

\section{SUMMARY AND DISCUSSION}

In summary, we presented a general scheme for realizing a quantum lock-in amplifier via many-body quantum interferometry under periodic modulations. On the basis of our protocol, the frequency and amplitude of an alternating signal can be efficiently extracted with a high signal-to-noise ratio, even in an extremely noisy environment. We analytically studied the measurement precisions for frequency and amplitude of an ac magnetic field via nonentangled and entangled many-body quantum states. For nonentangled states, the measurement precisions exhibit SQL scaling as expected. For entangled states such as spin cat states, by application of suitable interaction-based readout operations, the measurement precisions can achieve Heisenberg-limited scaling.

Moreover, we illustrated the robustness of our manybody quantum amplifier against stochastic noise. In the presence of stochastic noise, even for spin cat states, many-body quantum amplifiers can still achieve highprecision ac field sensing. Our scheme highlights the multi- $\pi$-pulse sequence as a useful technique for quantum sensing and provides a promising method for achieving Heisenberg-limited detection for alternating signals. 
(a) $|\mathrm{SCS}\rangle$

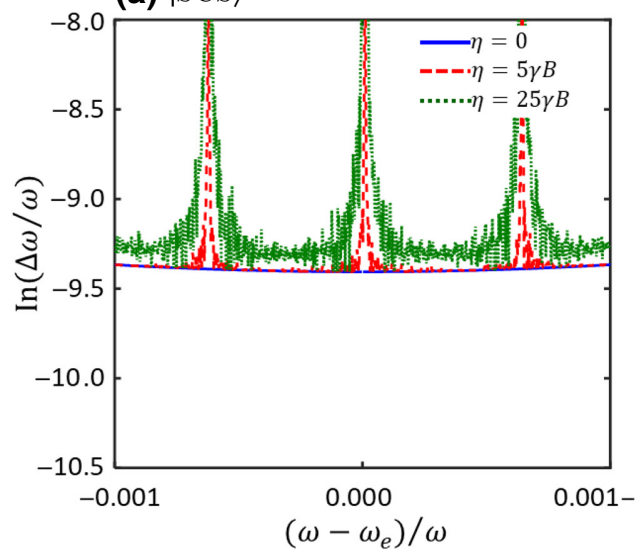

(b) $\mid$ cat $\rangle$

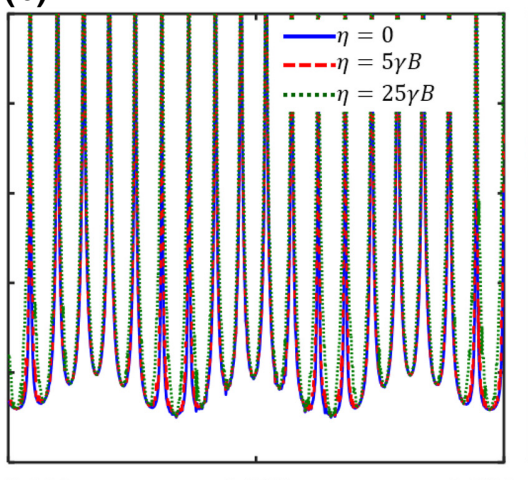

$\left(\omega-\omega_{e}\right) / \omega$ (c) $|\mathrm{GHZ}\rangle$

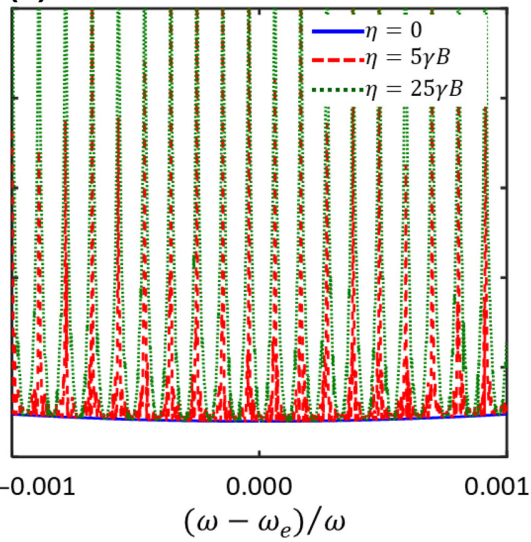

FIG. 5. The robustness of the many-body quantum amplifier against stochastic noise. The measurement precision $\Delta \omega$ versus versus the detuning $\omega-\omega_{e}$ for (a) the SCS, (b) the spin cat state $|\Psi(\theta=\pi / 8)\rangle_{\text {cat }}$, and (c) the GHZ state $|\Psi(\theta=0)\rangle_{\text {cat }}$. The solid blue lines are the results without stochastic noise $(\eta=0)$. The dashed red lines and the dotted green lines are the results under stochastic noise with $\eta=5 \gamma B$ and $\eta=25 \gamma B$, respectively. Here $T=2 \pi, \omega=20 \pi, \gamma B=2 \pi, N=6$, and $N_{o}(t) \in[-\eta$, $\eta]$. These results are averaged 20 times.

Meanwhile, compared with classical lock-in amplifiers, our scheme can realize different reference signals and resist different types of noise [70] via application of suitable periodic modulations.

In addition, our study may point to a new way for achieving time-dependent signal measurement via a manybody quantum lock-in amplifier. The amplifier demonstrates the power of the quantum lock-in measurement technique, which is readily available for various quantum probes. Single-particle quantum lock-in measurement techniques have been widely used for frequency metrology [20], magnetic field sensing [21,23,25], vector light shift detection [26], and force detection for the quantum motion of magnetic mechanical resonators [24]. By use of many-body quantum lock-in amplifiers, measurement precisions may be further increased.

To realize the entanglement-enhanced lock-in amplifier in experiments, one has to combine Ramsey interferometry with multiple rapid $\pi$ pulses in the interrogation stage. The precise implementation of $\pi$ pulses is a mature technology in quantum control $[7,11,15,17,20,21,71]$.

Furthermore, to achieve a Heisenberg-limited simultaneous measurement for frequency and amplitude, the preparation of the desired spin cat state and the implementation of an interaction-based readout are two key processes. Owing to the well-developed techniques in quantum control, various multiparticle entangled states have been generated in several systems, including nitrogenvacancy defect centers [72], Bose condensed atoms [12, 54,73-76], ultracold trapped ions [15,52], and solid-state spin systems [77]. In particular, for an ensemble of Bose condensed atoms occupying two hyperfine levels, it is feasible to prepare the desired spin cat state and achieve interaction-based readout via tuning the atom-atom interaction. In general, one can prepare the desired spin cat state via dynamical evolution $[12,54,73,74]$ or an adiabatic process $[29,31,32,43,75,76,78]$ under a one-axis twisting Hamiltonian $\hat{H}_{\text {twist }}=\chi \hat{J}_{z}^{2}+B_{x} \hat{J}_{x}$. The strength and the sign of the nonlinearity $\chi$, determined by the spatial overlap between different spin components and the $s$-wave scattering lengths, can be tuned via applying a spindependent force [74,79] or the techniques of Feshbach resonance $[12,74,80]$. Based on state-of-the-art techniques, our study is beneficial for the development of practical entanglement-enhanced quantum technologies, even in noise, such a, atomic clocks [81,82], magnetometers $[73,74,79,80,83]$, and weak-force detectors.

\section{ACKNOWLEDGMENTS}

The authors thank M. Jiang for helpful discussions. This work was supported by the National Natural Science Foundation of China (Grants No. 12025509 and No. 11874434), the Key-Area Research and Development Program of Guangdong Province (Grant No. 2019B030330001), and the Science and Technology Program of Guangzhou (Grant No. 201904020024). M.Z. is partially supported by the National Natural Science Foundation of China (Grant No. 12047563). J.H. is partially supported by the Guangzhou Science and Technology Projects (Grant No. 202002030459).

\section{APPENDIX A: NONCOMMUTATIVE OPERATIONS IN A MANY-BODY QUANTUM LOCK-IN AMPLIFIER}

In this section, we illustrate the importance of the noncommutative nature in the quantum lock-in amplifier. 
Because the quantum lock-in amplifier is analogous to the classical lock-in amplifier, we introduce the basic theory of the classical lock-in amplifier first, and then illustrate the basis of the quantum lock-in amplifier. In general, the target signal in the classical lock-in amplifier can be written as

$$
V(t)=V_{0} \sin \omega t
$$

and the reference signal is

$$
V_{R}(t)=\sin \omega_{e} t
$$

The classical lock-in amplifier generates the output signal via a mix-down process, i.e., $V(t)$ is multiplied by $V_{R}(t)$ and then integrated over an integration time $T$. Thus, the output signal is

$$
\begin{aligned}
I & =\int_{0}^{T} V(t) V_{R}(t) d t \\
& =\frac{V_{0}}{2 T} \int_{0}^{T}\left\{\cos \left[\left(\omega-\omega_{e}\right) t\right]-\cos \left[\left(\omega+\omega_{e}\right) t\right]\right\} d t .
\end{aligned}
$$

It is obviously that if the two frequencies are different, the average is zero. If the two frequencies are equal, the average value is equal to half of the target signal amplitude. Meanwhile, if the noise spectral components have frequencies far from the frequency of the reference signal, the negative effect will be averaged out in the integration. The mix-down process is essential for the classical lock-in amplifier, which can be achieved with a nonlinear device and generates the instantaneous product of the target signal and the reference signal. The key to realizing a quantum lock-in amplifier is finding a quantum analog of the mix-down process. Nonlinear dynamics of the wave function cannot be introduced directly, due to the linearity of the Schrödinger equation. However, the wave-function dynamics will be proportional to a product of Hamiltonian terms if the total Hamiltonian does not commute with itself at different times, and can be used to achieve a quantum mix-down process. Therefore, noncommuting dynamical manipulations are the basis of the quantum lock-in amplifier. In the main text, we choose $\hat{J}_{z}$ as the signal term, which is generally chosen in quantum metrology [12,84], such as in magnetic field measurement $[21,23,25,83]$ and frequency measurement $[20,81,82]$. The noncommuting dynamics is the key to the quantum lock-in technique. For the signal term $\hat{J}_{z}$, one can choose $\hat{J}_{x}, \hat{J}_{y}$, or other operators that do not commute with $\hat{J}_{z}$ as the mixing term. For simplicity, we choose $\hat{J}_{x}$ as the mixing term in the main text. In the field of quantum control, DD is a well-developed technique, and the sequence of $\pi$ pulses is ideal for realizing the mixing modulation.

\section{APPENDIX B: TIME EVOLUTION IN THE INTERACTION PICTURE}

In this section, we give the proof of Eqs. (2) and (6). The Schrödinger equation for an ensemble of two-level bosonic particles evolving under the Hamiltonian $\hat{H}=M(t) \hat{J}_{z}+$ $\Omega(t) \hat{J}_{x}$ can be written as

$$
i \frac{\partial|\Psi(t)\rangle}{\partial t}=\left[M(t) \hat{J}_{z}+\Omega(t) \hat{J}_{x}\right]|\Psi(t)\rangle .
$$

For convenience, we move into the interaction picture with respect to $\hat{H}_{\text {mix }}=\Omega(t) \hat{J}_{x}$. In this case, we have $|\Psi(t)\rangle=e^{-i \int_{0}^{t} \hat{H}_{\text {mix }}\left(t^{\prime}\right) d t^{\prime}}|\Psi(t)\rangle_{I}$, and thus the state evolution for $|\Psi(t)\rangle_{I}$ can be expressed as $(\hbar=1)$

$$
\begin{aligned}
i \frac{\partial|\Psi(t)\rangle_{I}}{\partial t} & =e^{i \int_{0}^{t} \hat{H}_{\text {mix }}\left(t^{\prime}\right) d t^{\prime}}\left[M(t) \hat{J}_{z}\right] e^{-i \int_{0}^{t} \hat{H}_{\text {mix }}\left(t^{\prime}\right) d t^{\prime}}|\Psi(t)\rangle_{I} \\
& =e^{i \int_{0}^{t} \Omega(t) \hat{J}_{x}\left(t^{\prime}\right) d t^{\prime}}\left[M(t) \hat{J}_{z}\right] e^{-i \int_{0}^{t} \Omega(t) \hat{S}_{x}\left(t^{\prime}\right) d t^{\prime}}|\Psi(t)\rangle_{I} .
\end{aligned}
$$

Defining $\alpha=\int_{0}^{t} \Omega\left(t^{\prime}\right) d t^{\prime}$, and since $\left[\hat{J}_{a}, \hat{J}_{b}\right]=i \epsilon_{a b c} \hat{J}_{c}\left(\epsilon_{a b c}\right.$ is the Levi-Civita symbol, with $a, b, c=x, y, z)$ and $e^{i \beta \hat{J}_{x}} \hat{J}_{z} e^{-i \beta \hat{J}_{x}}=\cos (\beta) \hat{J}_{z}+\sin (\beta) \hat{J}_{y}$, we have

$$
i \frac{\partial|\Psi(t)\rangle_{I}}{\partial t}=M(t) \cos (\alpha) \hat{J}_{z}+\sin (\alpha) \hat{J}_{y}|\Psi(t)\rangle_{I} .
$$

After integration, at time $T$, the evolved state $|\Psi(T)\rangle_{I}$ becomes

$$
\begin{aligned}
|\Psi(T)\rangle_{I} & =\hat{\mathcal{T}} e^{-i \int_{0}^{T} M(t)\left(\cos (\alpha) \hat{J}_{z}+\sin (\alpha) \hat{J}_{y}\right) d t}|\Psi(0)\rangle_{I} \\
& =\hat{\mathcal{T}} e^{\hat{A}}|\Psi(0)\rangle_{I}
\end{aligned}
$$

where $\hat{\mathcal{T}}$ is the time-ordering operator and the operator $\hat{A}$ can be written as

$$
\begin{aligned}
\hat{A} & =-i \int_{0}^{T} M(t) \cos (\alpha) \hat{J}_{z}+M(t) \sin (\alpha) \hat{J}_{y} d t \\
& =-i \int_{0}^{T} M(t) \cos \alpha d t \hat{J}_{z}+i \int_{0}^{T} M(t) \sin \alpha d t \hat{J}_{y} \\
& =-i\left(\varphi_{1} \hat{J}_{z}+\varphi_{2} \hat{J}_{y}\right) .
\end{aligned}
$$

Thus, we have

$$
|\Psi(T)\rangle_{I}=\hat{\mathcal{T}} e^{-i\left(\varphi \hat{J}_{z}+\varphi_{2} \hat{J}_{y}\right)}|\Psi(0)\rangle_{I},
$$

with $|\Psi(0)\rangle_{I}=|\Psi(0)\rangle$ and the two phase factors

$$
\begin{aligned}
\varphi_{1} & =\int_{0}^{T} M(t) \cos \alpha d t \\
& =\int_{0}^{T}\left[S_{0} \sin (\omega t+\beta)+N_{o}(t)\right] \cos \alpha d t
\end{aligned}
$$


and

$$
\begin{aligned}
\varphi_{2} & =\int_{0}^{T} M(t) \sin \alpha d t \\
& =\int_{0}^{T}\left[S_{0} \sin (\omega t+\beta)+N_{o}(t)\right] \sin \alpha d t .
\end{aligned}
$$

If one adds a suitable modulation $\Omega(t)$ to make $\cos \alpha$ and $\sin \alpha$ periodic and synchronized with signal $S(t)$, the phase accumulated owing to $S(t)$ adds up coherently, whereas the phase accumulated owing to $N_{o}(t)$ can average away.

\section{APPENDIX C: VALIDITY OF THE EFFECTIVE HAMILTONIAN}

In the interaction picture, the Hamiltonian for the interrogation stage reads

$$
\hat{H}_{I}=h(t)(\gamma B \sin \omega t) \hat{J}_{z},
$$

where $h(t)$ is the square wave function. When $\Omega(t)=$ $\pi \sum_{m=1}^{L} \delta(t-(m+1 / 2) \tau)$, the corresponding square wave function is

$$
h(t)=\left\{\begin{array}{rlll}
1 & \omega_{e} t+\pi / 2 & \bmod & 2 \pi \in[0, \pi), \\
-1 & \omega_{e} t+\pi / 2 & \bmod & 2 \pi \in[\pi, 2 \pi) .
\end{array}\right.
$$

Under the condition $\left|\omega-\omega_{e}\right| \ll \omega$ and $T \gg 2 \pi / \omega$, the Hamiltonian $(\mathrm{C} 1)$ can be approximated by an effective Hamiltonian $[23,25]$ :

$$
\hat{H}_{I}^{\mathrm{eff}}=\frac{2 \gamma B}{\pi} \sin \left[\left(\omega-\omega_{e}\right) t\right] \hat{J}_{z} .
$$

To compare the two Hamiltonians, we denote the integrals over time as

$$
\int_{0}^{T} \hat{H}_{I}=\Phi_{1} \hat{J}_{z}, \quad \int_{0}^{T} \hat{H}_{I}^{\mathrm{eff}}=\Phi_{2} \hat{J}_{z}
$$

where $\Phi_{1}$ and $\Phi_{2}$ are two phases dependent on frequency $\omega$ and amplitude $B$, which can be expressed as

$$
\begin{aligned}
& \Phi_{1}=\int_{0}^{T} h(t)(\gamma B \sin \omega t) d t, \\
& \Phi_{2}=\int_{0}^{T} \frac{2 \gamma B}{\pi} \sin \left[\left(\omega-\omega_{e}\right) t\right] d t .
\end{aligned}
$$

We confirm the validity of the effective Hamiltonian (C2) via numerical calculations. According to our numerical results, the two phases are almost the same when $\left(\left|\omega-\omega_{e}\right| / \omega\right) \leq 0.1$, i.e., $\Phi_{1} \approx \Phi_{2}$, for evolution time $T=$ $2 \pi$ and $\omega \geq 10 \pi$, as shown in Fig. 6 .

\section{APPENDIX D: VALIDITY OF THE ANALYTIC RESULTS IN THE INTERACTION PICTURE}

To illustrate the validity of the analytic results in the main text. We compare the numerical results obtained from the Schrödinger picture and the interaction picture with the analytic results obtained from the effective Hamiltonian. In our calculations, we plot the variation of the population difference versus time in the Schrödinger picture and the interaction picture and according to the analytic results for different values of $\tau$, as shown in Figs. 7 and 8. It is obvious that the analytic results are consistent with the numerical simulation from the Schrödinger picture as well as the interaction picture. This confirms that the results obtained from interaction picture are valid and the corresponding analytic results from the effective Hamiltonian are reasonable.

\section{APPENDIX E: INFLUENCE OF PULSE NUMBER}

Here we discuss the influence of the pulse number in our scheme. Since we work in the interaction picture, the
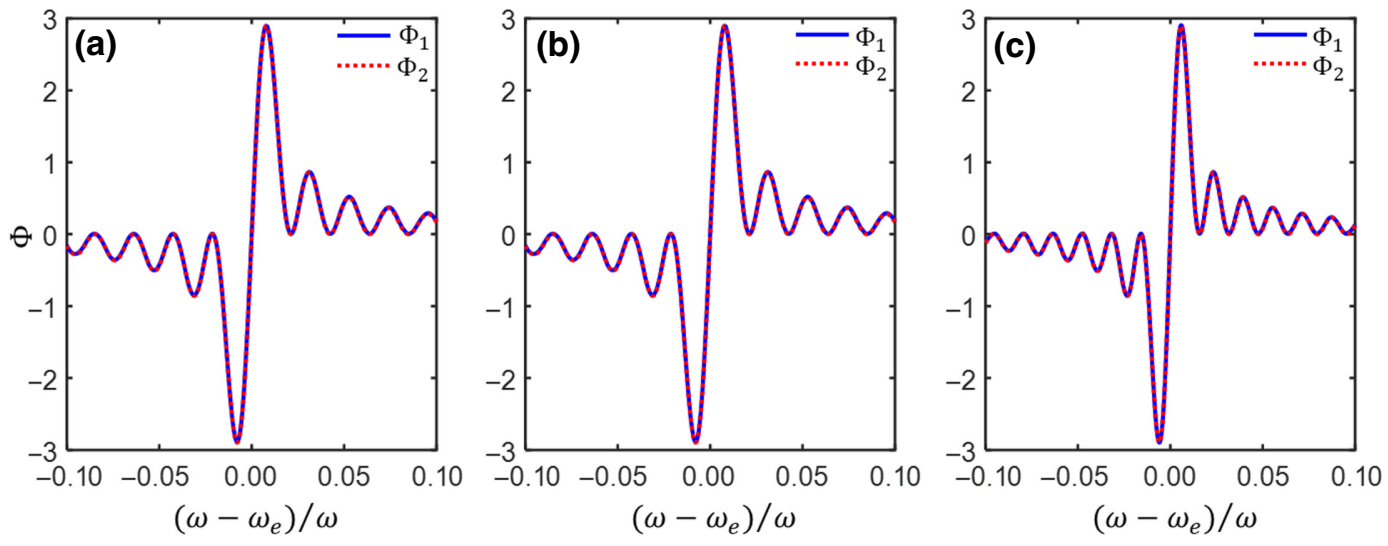

FIG. 6. Variations of the two phases $\Phi_{1}$ and $\Phi_{2}$ versus $\omega-\omega_{e}$ for (a) $\omega=10 \pi$, (b) $\omega=15 \pi$, and (c) $\omega=20 \pi$. Here $T=2 \pi$ and $\gamma B=1$. 


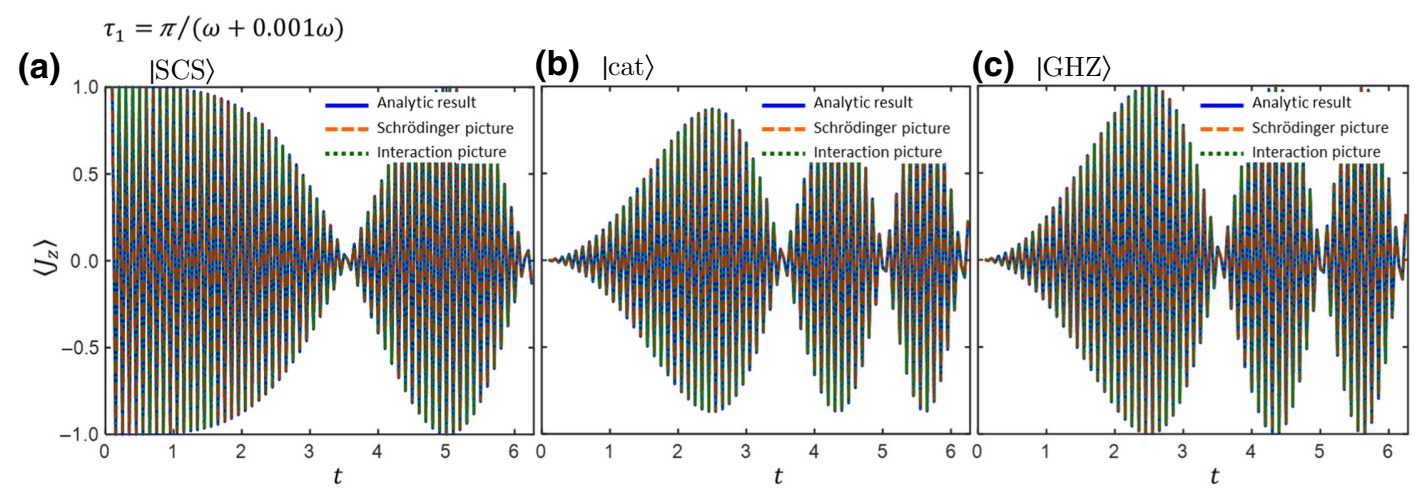

FIG. 7. Variation of half-population difference measurement versus time in the Schrödinger picture,in the interaction picture, and according to the analytic results. (a) Individual particles in the SCS, (b) the spin cat state $|\Psi(\theta=\pi / 8)\rangle_{\text {cat }}$, and (c) the GHZ state $|\Psi(\theta=0)\rangle_{\text {cat }}$. Here $\omega=20 \pi, \gamma B=2 \pi, N=2$, and $\tau_{1}=\pi /(\omega+0.001 \omega)$.

operator can be written as

$$
\hat{O}^{I}(t)=e^{i \alpha \hat{J}_{x}} \hat{O} e^{-i \alpha \hat{J}_{x}},
$$

where $\hat{O}$ is the operator in the Schrödinger picture. In our scheme, we consider the modulation is a set of sharp $\pi$ pulses and can be approximated as $\Omega(t)=\pi \sum_{m=1}^{L} \delta(t-$ $(m+1 / 2) \tau)$. Thus, at time $T$, the operator in the interaction picture can be written as

$$
\begin{aligned}
\hat{O}^{I}(T)= & e^{i \int_{0}^{T} \pi \sum_{m=1}^{L} \delta\left(t-\left(m+\frac{1}{2}\right) \tau\right) d \hat{t}_{x}} \hat{O} \\
& \times e^{-i \int_{0}^{T} \pi \sum_{m=1}^{L} \delta\left(t-\left(m+\frac{1}{2}\right) \tau\right) d \hat{t}_{x}} .
\end{aligned}
$$

When the evolution time $T$ is fixed, for the $i$ th pulse spacing $\tau_{i}$, the number of $\pi$ pulses is given by $L=$ $\left[T / \tau_{i}+1 / 2\right]$, where [-] stands for the integer-valued division. Because of the property $\int \delta(x) d x=1$, we have $\int_{0}^{T} \pi \sum_{m=1}^{L} \delta(t-(m+1 / 2) \tau) d t=L \pi$. Therefore, the operator in the interaction picture becomes

$$
\hat{O}^{I}(t)=e^{i L \pi \hat{J}_{x}} \hat{O} e^{-i L \pi \hat{J}_{x}} .
$$

For the collective spin operators, we have

$$
\begin{aligned}
& \hat{J}_{x}^{I}=e^{i L \pi \hat{J}_{x}} \hat{J}_{x} e^{-i L \pi \hat{J}_{x}}=\hat{J}_{x}, \\
& \hat{J}_{y}^{I}=e^{i L \pi \hat{J}_{x}} \hat{J}_{y} e^{-i L \pi \hat{J}_{x}}=\cos (L \pi) \hat{J}_{y}-\sin (L \pi) \hat{J}_{z}, \\
& \hat{J}_{z}^{I}=e^{i L \pi \hat{J}_{x}} \hat{J}_{z} e^{-i L \pi \hat{J}_{x}}=\cos (L \pi) \hat{J}_{z}+\sin (L \pi) \hat{J}_{y} .
\end{aligned}
$$

According to Eq. (E4), we find that the parity of the pulse number alters the operators. When $L$ is an even number, the collective spin operators in the interaction picture are

$$
\hat{J}_{x}^{I}=\hat{J}_{x}, \hat{J}_{y}^{I}=\hat{J}_{y}, \hat{J}_{z}^{I}=\hat{J}_{z} .
$$

While $L$ is an odd number, the operators become

$$
\hat{J}_{x}^{I}=\hat{J}_{x}, \hat{J}_{y}^{I}=-\hat{J}_{y}, \hat{J}_{z}^{I}=-\hat{J}_{z} .
$$

In contrast to $\hat{J}_{x}^{I}$, the operators $\hat{J}_{y}^{I}$ and $\hat{J}_{z}^{I}$ change sign when $L$ is odd. Thus, the half-population difference of the final

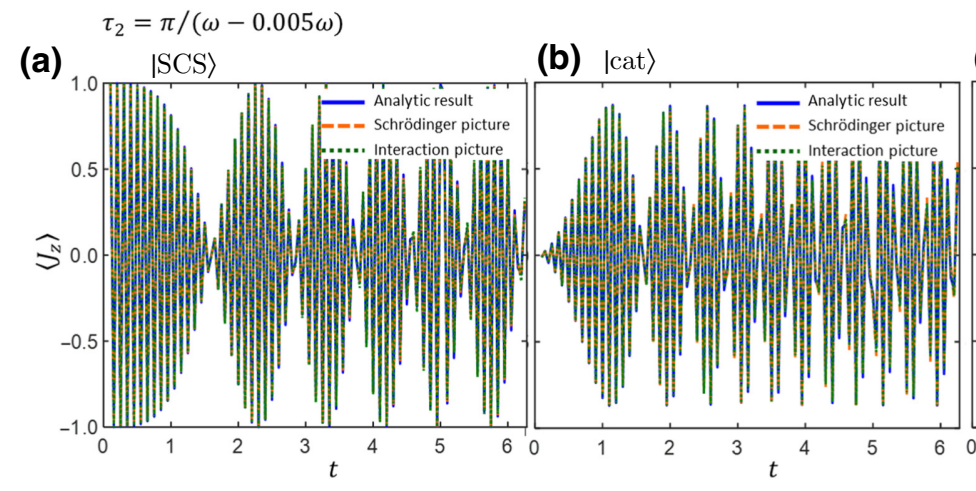

(c) $\mid \mathrm{GHZ}$

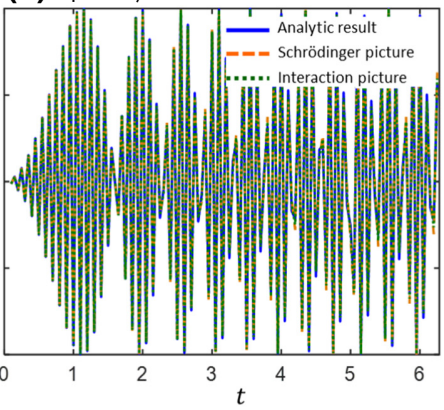

FIG. 8. Variation of half-population difference measurement versus time in the Schrödinger picture, in the interaction picture, and according to the analytic results. (a) Individual particles in the SCS, (b) the spin cat state $|\Psi(\theta=\pi / 8)\rangle_{\text {cat }}$, and (c) the GHZ state $|\Psi(\theta=0)\rangle_{\text {cat }}$. Here $\omega=20 \pi, \gamma B=2 \pi, N=2$, and $\tau_{1}=\pi /(\omega-0.005 \omega)$. 
(a)

(b)

(c)

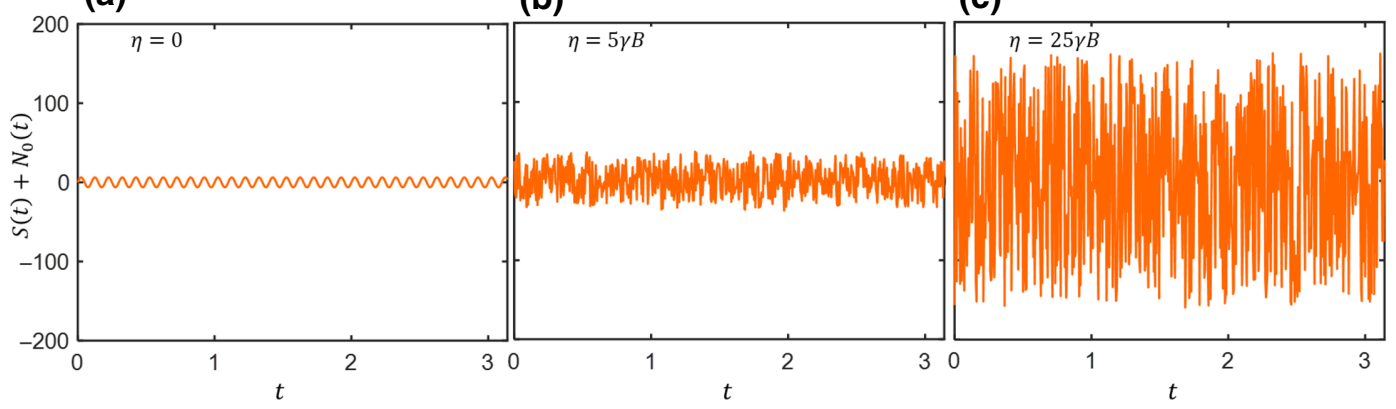

FIG. 9. Variation of the total signal versus time. (a) Total signal $S(t)+N_{o}(t)$ with (a) $\eta=0$, (b) $\eta=5 \gamma B$, and (c) $25 \gamma B$. Here $\omega=20 \pi$ and $N_{o}(t) \in[-\eta, \eta]$.

state $\left\langle\hat{J}_{z}\right\rangle_{f}$ is dependent on the parity of the pulse number $L$. According to Eq. (E2), we give the expression for the unitary operator $\hat{U}_{I}$ in the Schrödinger picture. For the unitary operator $\hat{U}_{I}=e^{-i(\pi / 2) \hat{J}_{y}}$, the corresponding expression in the Schrödinger picture can be written as

$$
\hat{U}=e^{-i L \pi \hat{J}_{x}} e^{-i \frac{\pi}{2} \hat{J}_{y}} e^{i L \pi \hat{J}_{x}}
$$

For the unitary operator $\hat{U}_{I}=e^{i(\pi / 2) \hat{J}_{x}} e^{i(\pi / 2) \hat{J}_{z}^{2}} e^{i(\pi / 2) \hat{J}_{x}}$, the corresponding expression in the Schrödinger picture can be written as

$$
\hat{U}=e^{-i\left(L-\frac{1}{2}\right) \pi \hat{J}_{x}} e^{i \frac{\pi}{2} \hat{J}_{z}^{2}} e^{i\left(L+\frac{1}{2}\right) \pi \hat{J}_{x}} .
$$

\section{APPENDIX F: THE TOTAL SIGNAL IN THE PRESENCE OF NOISE}

To demonstrate the robustness against stochastic noise of the many-body quantum lock-in amplifier, we study practical noisy signals. Without loss of generality, we assume $N_{o}(t)$ is the stochastic noise, $N_{o}(t) \in[-\eta, \eta]$, and its long-time integration $\overline{N_{o}(t)}=0$. Generally, the target signal is submerged in noise. As shown in Fig. 9, we plot the total signals $S(t)+N_{o}(t)$ with $\eta=0, \eta=5 \gamma B$, and $\eta=25 \gamma B$. (a)

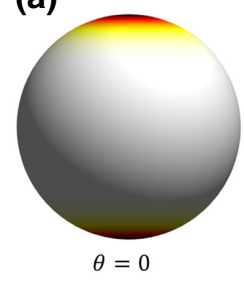

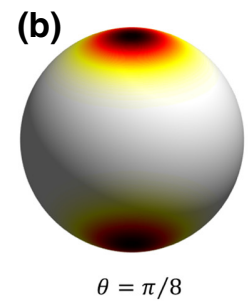

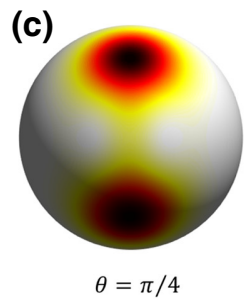

(c)

$\theta=\pi / 4$
FIG. 10. Husimi distributions for the spin cat state with different $\theta$. (a) Husimi distributions for (a) the GHZ state $|\Psi(\theta=0)\rangle_{\text {cat }}$, (b) the spin cat state $|\Psi(\theta=\pi / 8)\rangle_{\text {cat }}$, and (c) the spin cat state $|\Psi(\theta=\pi / 4)\rangle_{\text {cat }}$. Here $N=20$.

\section{APPENDIX G: SPIN CAT STATES}

Spin cat states are typical kinds of MSSCS [36,51]. Here we discuss MSSCS in the form

$$
|\Psi(\theta, \eta)\rangle_{M}=N_{c}(|\theta, \eta\rangle+|\pi-\theta, \eta\rangle)
$$

where $N_{c}$ is the normalization and $|\theta, \eta\rangle$ denotes the $N$ particle SCS:

$$
|\theta, \eta\rangle=\sum_{m=J}^{J} c_{m}^{J}(\theta) e^{-i(J+m) \eta}|J, m\rangle,
$$

where $\quad c_{m}^{J}(\theta)=\sqrt{[(2 J) ! /(J+m) !(J-m) !]} \cos ^{J+m}(\theta /$ 2) $\sin ^{J-m}(\theta / 2)$, and $|J, m\rangle$ represents the Dicke basis with $J=N / 2$ and $m=-J,-J+1, \ldots, J-1, J$. Without loss of generality, we assume $\eta=0$ and consider the states in the form

$$
\begin{aligned}
|\Psi(\theta)\rangle_{\mathrm{MSSCS}} & =N_{c}\left[|\Psi(\theta)\rangle_{\mathrm{SCS}}+|\Psi(\pi-\theta)\rangle_{\mathrm{SCS}}\right] \\
& =N_{c}\left\{\sum_{m=-J}^{J}\left[c_{m}^{J}(\theta)+c_{m}^{J}(\pi-\theta)\right]|J, m\rangle\right\} \\
& =N_{c}\left\{\sum_{m=-J}^{J} c_{m}^{J}(\theta)(|J, m\rangle+|J,-m\rangle)\right\} .
\end{aligned}
$$

Here the two SCSs have the same azimuthal angle $\eta=0$ and the polar angles are symmetric about $\theta=\pi / 2$. Since $C_{m}^{J}(\theta)=C_{m}^{J}(\pi-\theta)$, the coefficients of the MSSCS are symmetric about $m=0$. It was shown that when the two superposition SCSs are orthogonal or quasiorthogonal, the corresponding MSSCS can be regarded as a spin cat state [36,51]. The sufficient condition of spin cat states has been studied in detail [36,51]. Mathematically, the sufficient 
condition of spin cat states can be expressed as

$$
\theta \leq \theta_{c} \equiv \sin ^{-1}\left(2\left\{\frac{[(J-1) !]^{2}}{2(2 J) !}\right\}^{\frac{1}{2 J}}\right) .
$$

For total particle number $N \geq 6$, when $\theta \leq \pi / 8$, the corresponding MSSCS can be regarded as a spin cat state. Under this condition, we approximate the spin cat states as

$$
|\Psi(\theta)\rangle_{\mathrm{cat}}=\frac{1}{\sqrt{2}}\left[\sum_{m=-J}^{J} c_{m}^{J}(\theta)(|J, m\rangle+|J,-m\rangle)\right] .
$$

According to Eq. (G5), the spin cat states can be understood as a superposition of GHZ states with different spin length. Especially, when $\theta=0$, the spin cat state corresponds to the GHZ state. To illustrate the spin cat state clearly, we show the Husimi distributions for the spin cat state on the Bloch sphere in Fig. 10.

\section{APPENDIX H: VARIATION OF THE MEASUREMENT PRECISON}

In the main text, we show only the optimal measurement precision for the two parameters. In this section, we give the variation of the measurement precision with detuning $\omega-\omega_{e}$ and amplitude $B$ in detail. According to the analytic expression for the measurement precision, we find that the measurement precisions $\Delta \omega$ and $\Delta B$ are dependent on both the frequency $\omega$ and the amplitude $B$ simultaneously. For comparison, we choose three typical input states: the SCS $|\Psi\rangle_{\mathrm{SCS}}$, the spin cat state $|\Psi(\pi / 8)\rangle_{\text {cat }}$, and the GHZ state $|\Psi(0)\rangle_{\text {cat }}$. In Fig. 11, the variations of $\Delta \omega / \omega$ and $\gamma \Delta B / \omega$ versus $B$ and $\omega-\omega_{e}$ with the three input states are shown. When the input state is a SCS or a GHZ state, the measurement precision $\Delta \omega$ attains its optimal value when $\cos \left[\left(\omega-\omega_{e}\right) T\right][2-(\omega-$ $\left.\left.\omega_{e}\right)^{2} T^{2}\right]+2 T\left(\omega-\omega_{e}\right) \sin \left[\left(\omega-\omega_{e}\right) T\right]=2$ for fixed particle number $N$. Meanwhile, the measurement precision $\Delta B$ is dependent only on the frequency $\omega$. The measurement precision of $\Delta B$ attains its optimal value when $\cos \left[\left(\omega-\omega_{e}\right) T\right]+\left(\omega-\omega_{e}\right) T \sin \left[\left(\omega-\omega_{e}\right) T\right]=1$. In contrast, the dependence of $\Delta \omega / \omega$ and $\gamma \Delta B / \omega$ on $B$ and $\omega-\omega_{e}$ with spin cat states $|\Psi(\theta)\rangle_{\text {cat }}(\theta \neq 0)$ is more complicated; see, for example, Figs. 11(b) and 11(e).

\section{APPENDIX I: MEASUREMENT PRECISIONS IN TWO SPECIAL SITUATIONS FOR SPIN CAT STATES}

For a spin cat state, we give the general expressions for the measurement precisions $\Delta \omega$ and $\Delta B$ in the main text. Here we discuss measurement precisions in two special situations $(\phi \rightarrow 0$ and $\phi=\pi / 2)$ for the spin cat state in detail.

When $\omega-\omega_{e} \rightarrow 0, \phi=(2 \gamma B / \pi)\left(1-\cos \left[\left(\omega-\omega_{e}\right) T\right]\right) /$ $\left(\omega-\omega_{e}\right) \rightarrow 0, \sin 2 m \pi \approx 0$, and $\cos 2 m \pi \approx 1$, the measurement precisions $\Delta \omega$ and $\Delta B$ can be simplified as

$$
\begin{aligned}
\left.\Delta \omega\right|_{\phi=0} & \approx \frac{\pi}{2 \gamma B} \frac{\sqrt{\sum_{m=-J}^{m=J} m^{2}\left|c_{m}^{J}(\theta)\right|^{2}}}{\left.\left|2 \sum_{m=-J}^{m=J} m^{2}(-1)^{J-m}\right| c_{m}^{J}(\theta)\right|^{2} \mid} \frac{\left(\omega-\omega_{e}\right)^{2}}{|G|}=\tilde{B}(J, \theta) \frac{\pi}{2 \gamma B} \frac{\left(\omega-\omega_{e}\right)^{2}}{|G|} \\
& =\tilde{B}(J, \theta) \frac{\pi}{3 \gamma B T^{2}}, \\
\left.\Delta B\right|_{\phi=0} & \approx \frac{\sqrt{\sum_{m=-J}^{m=J} m^{2}\left|c_{m}^{J}(\theta)\right|^{2}}}{\left.\left|2 \sum_{m=-J}^{m=J} m^{2}(-1)^{J-m}\right| c_{m}^{J}(\theta)\right|^{2} \mid} \frac{\pi}{2 \gamma}\left|\frac{\omega-\omega_{e}}{\cos \left[\left(\omega-\omega_{e}\right) T\right]-1}\right| \\
& =\tilde{B}(J, \theta) \frac{\pi}{2 \gamma} \frac{\omega-\omega_{e}}{\cos \left[\left(\omega-\omega_{e}\right) T\right]-1}=\tilde{B}(J, \theta) \frac{\pi}{\gamma\left(\omega-\omega_{e}\right) T^{2}} .
\end{aligned}
$$

When $\phi=(2 \gamma B / \pi)\left\{\left(1-\cos \left[\left(\omega-\omega_{e}\right) T\right]\right) /\left(\omega-\omega_{e}\right)\right\}=(\pi / 2), \sin m \pi=0$, and $(-1)^{J-m} \cos m \pi=1$, the measurement precisions $\Delta \omega$ and $\Delta B$ can be simplified as

$$
\begin{aligned}
& \left.\Delta \omega\right|_{\phi=\frac{\pi}{2}}=\frac{\pi}{2 \gamma B} \frac{\sqrt{\sum_{m=-J}^{m=J} m^{2}\left|c_{m}^{J}(\theta)\right|^{2}}}{\left.\left|2 \sum_{m=-J}^{m=J} m^{2}\right| c_{m}^{J}(\theta)\right|^{2} \mid} \frac{\left(\omega-\omega_{e}\right)^{2}}{|G|}=C(\theta) \frac{\pi}{2 \gamma B N} \frac{\left(\omega-\omega_{e}\right)^{2}}{|G|}, \\
& \left.\Delta B\right|_{\phi=\frac{\pi}{2}}=\frac{\sqrt{\sum_{m=-J}^{m=J} m^{2}\left|c_{m}^{J}(\theta)\right|^{2}}}{\left.\left|2 \sum_{m=-J}^{m=J} m^{2}\right| c_{m}^{J}(\theta)\right|^{2} \mid} \frac{\pi}{2 \gamma}\left|\frac{\omega-\omega_{e}}{\cos \left[\left(\omega-\omega_{e}\right) T\right]-1}\right|=C(\theta) \frac{\pi}{2 \gamma N}\left|\frac{\omega-\omega_{e}}{\cos \left[\left(\omega-\omega_{e}\right) T\right]-1}\right| .
\end{aligned}
$$




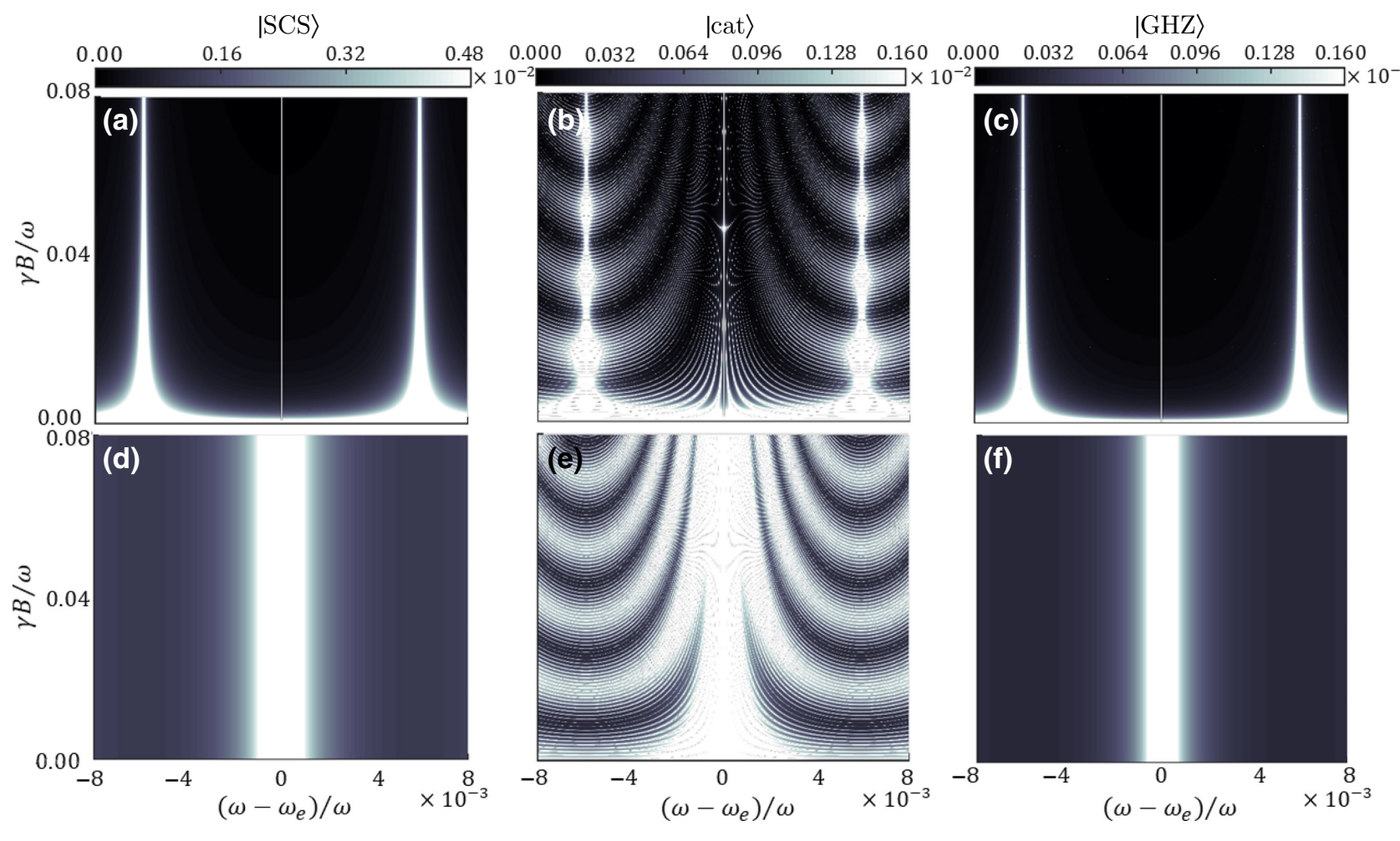

FIG. 11. Variation of measurement precisions for individual particles and entangled particles for fixed $N$. (a) $\Delta \omega / \omega$ and (d) $\gamma \Delta B / \omega$ for a SCS with $\hat{U}_{I}=e^{-i \frac{\pi}{2} \hat{y}_{y}}$. (b) $\Delta \omega / \omega$ and (e) $\gamma \Delta B / \omega$ for a spin cat state with $\hat{U}_{I}=e^{i \frac{\pi}{2} \hat{J}_{x}} e^{i \frac{\pi}{2} \hat{J}_{z}^{2}} e^{i \frac{\pi}{2} \hat{J}_{x}}$ and $\theta=\pi / 8$. (c) $\Delta \omega / \omega$ and (f) $\gamma \Delta B / \omega$ for a GHZ state with $\hat{U}_{I}=e^{i \frac{\pi}{2} \hat{J}_{x}} e^{i \frac{\pi}{2} \hat{J}_{z}^{2}} e^{i \frac{\pi}{2} \hat{J}_{x}}$. Here $T=2 \pi, \omega=20 \pi$, and $N=20$.

The coefficient $C(\theta)=1+\left[2 \tan ^{2}(\theta / 2) /\left(1+\tan ^{2}(\theta / 2)\right)\right]$ is independent of the particle number $N$ [51]. According to Eqs. (I3) and (I4), it is obvious that the measurement precisions $\Delta \omega$ and $\Delta B$ both can approach Heisenberg-limited scaling.

\section{APPENDIX J: INFLUENCE OF INITIAL PHASE SHIFT BETWEEN THE INPUT AND REFERENCE SIGNALS}

In our scheme, the modulation of $\Omega(t)$ is a set of $\pi$ pulses in the form of $\Omega(t)=\pi \sum_{m=1}^{L} \delta(t-[m+$ $(1+k / 2)] \tau)$, where $k \in[-1,1)$. The parameter $k$ corresponds to the initial phase shift between the input and reference signals. The lock-in case of $k=0$ has been discussed. In the following, we consider the other condition, where $k=-1$.

The Hamiltonian for the interrogation stage (after moving to the interaction picture with respect to the modulation) reads

$$
\hat{H}_{I}=h(t)[\gamma B \sin (\omega t)] \hat{J}_{z},
$$

where the square wave function $h(t)$ becomes

$$
h(t)=\left\{\begin{array}{rlll}
1 & \omega_{e} t & \bmod & 2 \pi \in[0, \pi), \\
-1 & \omega_{e} t & \bmod & 2 \pi \in[\pi, 2 \pi) .
\end{array}\right.
$$

Similarly, one can easily tune $\omega_{e}$ close to $\omega$ in an experiment. When $\left|\omega-\omega_{e}\right| \ll \omega$ and $T \gg 2 \pi / \omega$, the Hamiltonian $(\mathrm{J} 1)$ can be approximated by an effective Hamiltonian (the approximation is also valid for $\left|\omega-\omega_{e}\right| / \omega<0.1$ when $\omega>10 \pi$ ):

$$
\hat{H}_{I}^{\mathrm{eff}}=\frac{2 \gamma B}{\pi} \cos \left[\left(\omega-\omega_{e}\right) t\right] \hat{J}_{z} .
$$

After similar procedures as in the case of $k=1$, the final state before half-population difference measurement can be written as

$$
|\Psi\rangle_{\text {final }}=\hat{U} e^{-i \alpha \hat{J}_{x}} e^{-i \phi \hat{J}_{z}}|\Psi\rangle_{\text {in }} .
$$

The analytic form of the phase is written as

$$
\phi_{1}=\frac{2 \gamma B}{\pi} \frac{\sin \left[\left(\omega-\omega_{e}\right) T\right]}{\omega-\omega_{e}} .
$$

We first consider individual particles without any entanglement and then discuss the case of spin cat states. For the spin coherent state $|\Psi\rangle_{\mathrm{SCS}}$, the final state before the half-population difference measurement can be written as

$$
|\Psi\rangle_{\text {final }}^{I}=e^{-i \frac{\pi}{2} \hat{J}_{y}} e^{-i \phi_{1} \hat{J}_{z}}|\Psi\rangle_{\mathrm{SCS}} .
$$

In an explicit form, the final state becomes 


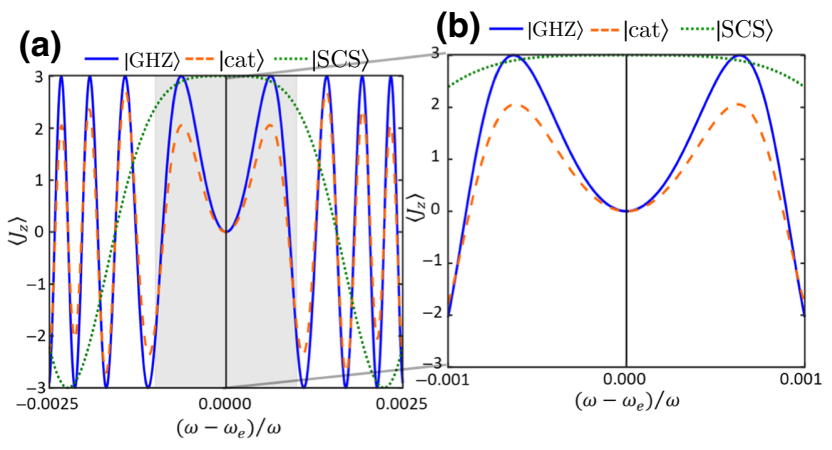

FIG. 12. Variation of the measurement signal $J_{z}$ versus detuning $\omega-\omega_{e}$ for individual particles and entangled particles for fixed $B$. The dotted green line show the results for a SCS with $\hat{U}_{I}=e^{-i \frac{\pi}{2} \hat{J}_{y}}$. The dashed orange line shows the results for a spin cat state with $\hat{U}_{I}=e^{i \frac{\pi}{2} \hat{J}_{x}} e^{i \frac{\pi}{2} \hat{J}_{z}^{2}} e^{i \frac{\pi}{2} \hat{J}_{x}}$ and $\theta=\pi / 8$. The solid blue line show the results for a GHZ state with $\hat{U}_{I}=e^{i \frac{\pi}{2} \hat{J}_{x}} e^{i \frac{\pi}{2} \hat{J}_{z}^{2}} e^{i \frac{\pi}{2} \hat{J}_{x}}$. These results indicate that the expectation of the half-population difference is symmetric for detuning $\omega-\omega_{e}$ and the lock-in point located at $\omega-\omega_{e}=0$, i.e., $\omega=\omega_{e}$. Here $T=2 \pi, \omega=20 \pi$, $\gamma B=2 \pi, N=6$, and $k=0$.

$$
|\Psi\rangle_{\text {final }}^{I}=\sum_{m=-J}^{J} \sqrt{C_{J}^{m}}\left(-\cos \frac{\phi_{1}}{2}\right)^{J+m}\left(i \sin \frac{\phi_{1}}{2}\right)^{J-m}|J, m\rangle
$$

where $C_{J}^{m}=[(2 J) ! /(J+m) !(J-m) !]$ is the binomial coefficient. After some algebra, the expectations of the half-population difference and the square of the halfpopulation difference on the final state can be explicitly written as

$$
\begin{aligned}
\left\langle J_{z}\right\rangle_{f} & =(-1)^{L} \frac{N}{2} \cos \phi_{1}, \\
\left\langle J_{z}^{2}\right\rangle_{f} & =\frac{N}{4}+\frac{N(N-1)}{4} \cos ^{2} \phi_{1} .
\end{aligned}
$$

Thus, the measurement signal $J_{z}$ is given by

$$
J_{z}=\frac{N}{2} \cos \phi_{1}
$$

From Eq. (J8), it is found that the information for the estimated parameters $\omega$ and $B$ can be inferred from the bisinusoidal oscillation of the measurement signal $J_{z}$. In our calculation, we find Eq. (J8) with respect to detuning $\omega-\omega_{e}$ is symmetric for fixed $B$ and the lock-in point located at $\omega-\omega_{e}=0$, i.e., $\omega=\omega_{e}$, as shown in Fig. 12 (dotted green line). Thus, one can obtain the value of $\omega$, and further extract the value of $B$ according to the analytic form of Eq. (J8). This implies that the values of $\omega$ and $B$ can be simultaneously obtained by means of only the measurement signal $J_{z}$.

According to the quantum estimation theory [1,57-59], the measurement precisions of the estimated parameters can be given according to the error propagation formula. The measurement precisions for $\omega$ and $B$ are

$$
\Delta \omega=\frac{\left(\Delta \hat{J}_{z}\right)_{f}}{\left|\partial\left\langle\hat{J}_{z}\right\rangle_{f} / \partial \omega_{e}\right|}
$$

and

$$
\Delta B=\frac{\left(\Delta \hat{J}_{z}\right)_{f}}{\left|\partial\left\langle\hat{J}_{z}\right\rangle_{f} / \partial B\right|}
$$

where

$$
\left(\Delta \hat{J}_{z}\right)_{f}=\sqrt{\left\langle\hat{J}_{z}^{2}\right\rangle_{f}-\left\langle\hat{J}_{z}\right\rangle_{f}^{2}}
$$

Substituting Eqs. (J6) and (J7) into Eqs. (J9) and (J10), one can analytically obtain the measurement precisions $\Delta \omega$ and $\Delta B$. They read

$$
\begin{aligned}
\Delta \omega & =\frac{\pi}{2 \gamma B \sqrt{N}} \frac{\left(\omega-\omega_{e}\right)^{2}}{\sin \left[\left(\omega-\omega_{e}\right) T\right]-T\left(\omega-\omega_{e}\right) \cos \left[\left(\omega-\omega_{e}\right) T\right] \mid}, \\
\Delta B & =\frac{\pi}{2 \gamma \sqrt{N}}\left|\frac{\omega-\omega_{e}}{\sin \left[\left(\omega-\omega_{e}\right) T\right]}\right| .
\end{aligned}
$$

From Eqs. (J12) and (J13), the measurement precisions $\Delta \omega$ and $\Delta B$ exhibit only SQL scaling. For fixed $N$, the measurement precision $\Delta \omega$ is dependent on both the detuning $\omega-\omega_{e}$ and $B$, as shown in Fig. 13(a). When $\sin \left[\left(\omega-\omega_{e}\right) T\right]\left[2-\left(\omega-\omega_{e}\right)^{2} T^{2}\right]-$ $2 T\left(\omega-\omega_{e}\right) \cos \left[\left(\omega-\omega_{e}\right) T\right]=0, \Delta \omega$ attains the optimal value. However, the measurement precision $\Delta B$ is dependent only on the detuning $\omega-\omega_{e}$, as shown in Fig. 13(d). When it is near resonance $\omega \approx \omega_{e}, \Delta B=\pi / 2 \gamma \sqrt{N} T$ is optimal.

We numerically calculate the optimal measurement precisions versus particle number in the range $\left|\left(\omega-\omega_{e}\right) / \omega\right| \epsilon$ 

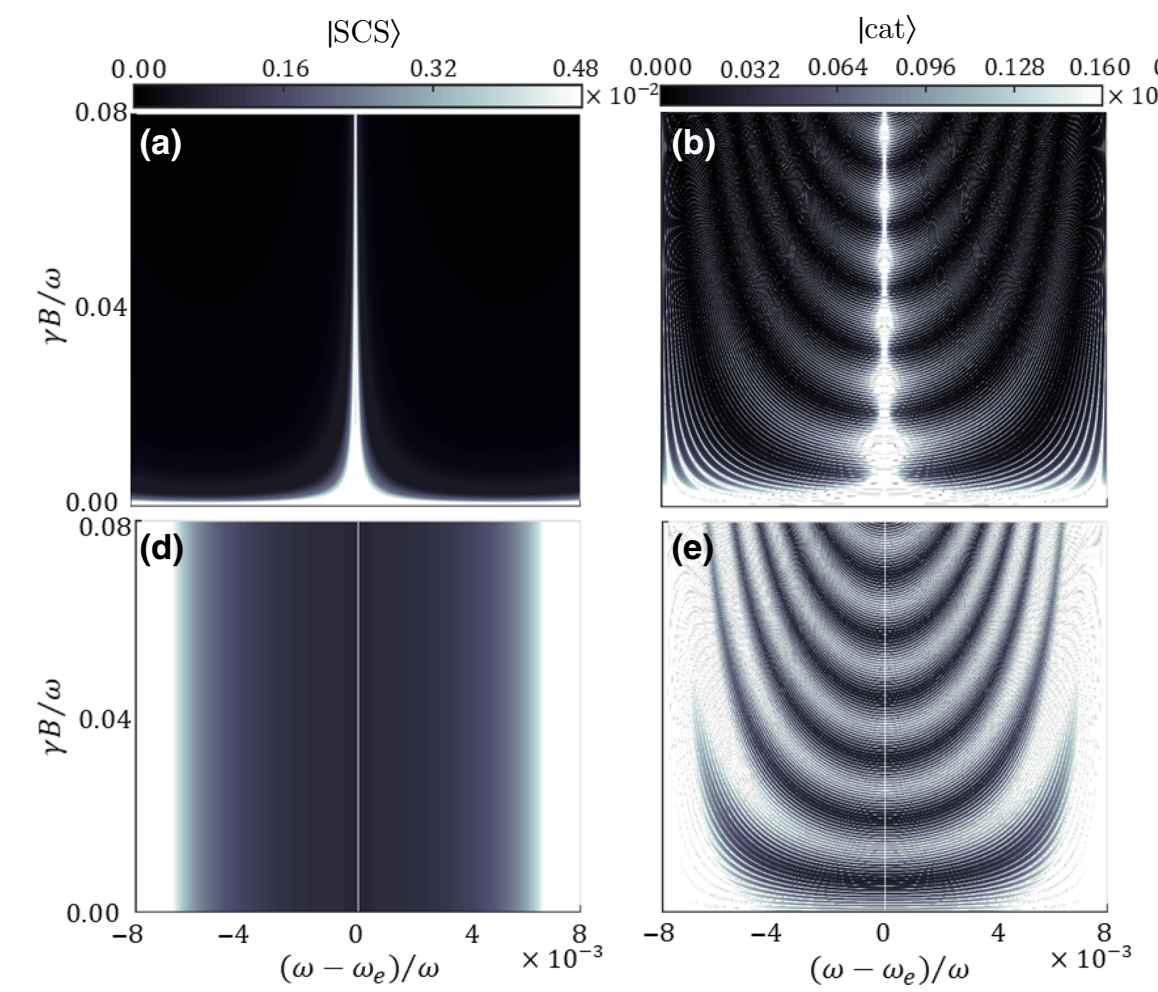

|GHZ〉
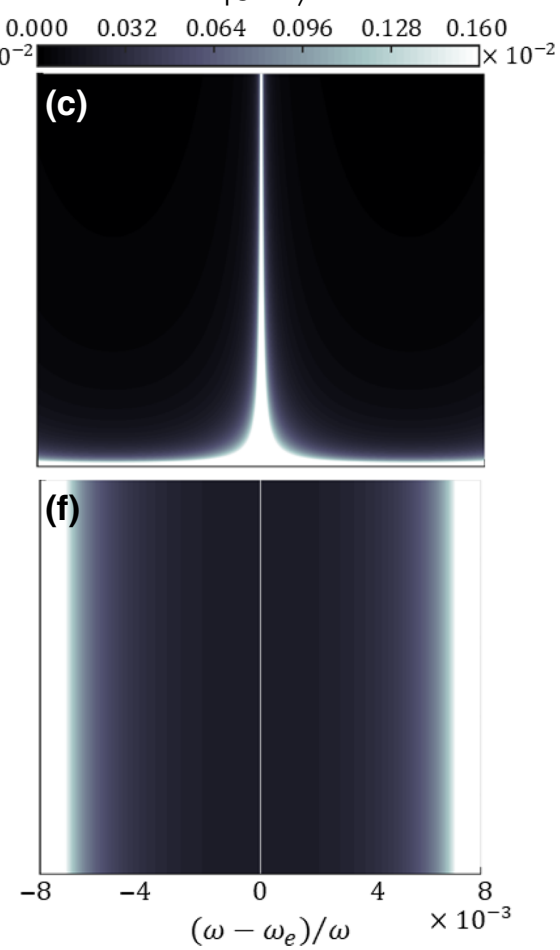

FIG. 13. Variation of the measurement precisions for individual particles and entangled particles for fixed $N$. (a) $\Delta \omega / \omega$ and(d) $\gamma \Delta B / \omega$ for a SCS with $\hat{U}_{I}=e^{-i \frac{\pi}{2} \hat{J}_{y}}$. (b) $\Delta \omega / \omega$ and (e) $\gamma \Delta B / \omega$ for a spin cat state with $\hat{U}_{I}=e^{i \frac{i}{2} \hat{J}_{x}} e^{i \frac{\pi}{2} \hat{J}_{z}^{2}} e^{i \frac{\pi}{2} \hat{J}_{x}}$ and $\theta=\pi / 8$. (c) $\Delta \omega / \omega$ and (f) $\gamma \Delta B / \omega$ for a GHZ state with $\hat{U}_{I}=e^{i \frac{\pi}{2} \hat{J}_{x}} e^{i \frac{\pi}{2} \hat{J}_{z}^{2}} e^{i \frac{\pi}{2} \hat{J}_{x}}$. Here $T=2 \pi, \omega=20 \pi, N=20$, and $k=0$.

$[0,0.008]$ and for $\gamma B=2 \pi$. According to the fitting results (see Fig. 14), the log-log measurement precisions $\ln \left(\Delta \omega_{\min } / \omega\right) \approx-0.5 \ln N-8.37$ and $\ln \left(\gamma \Delta B_{\min } / \omega\right) \approx$ $-0.5 \ln N-5.53$. For the two parameters, the optimal measurement precisions $\Delta \omega_{\min }$ [purple circles in Fig. 14(a)] and $\Delta B_{\min }$ [purple circles in Fig. 14(b)] both exhibit SQL scaling as expected.

Next we try to use the spin cat states to perform the measurement. We choose an interaction-based operation $\hat{U}_{I}=$ $\hat{U}=e^{i(\pi / 2) \hat{J}_{x}} e^{i(\pi / 2) \hat{J}_{z}^{2}} e^{i(\pi / 2) \hat{J}_{x}}$ in the readout stage. Therefore, the final state before the half-population difference can be written as

$$
|\Psi\rangle_{\text {final }}^{I}=e^{i \frac{\pi}{2} \hat{J}_{x}} e^{i \frac{\pi}{2} \hat{J}_{z}^{2}} e^{i \frac{\pi}{2} \hat{J}_{x}} e^{-i \phi_{1} \hat{J}_{z}}|\Psi(\theta)\rangle_{\text {cat }} .
$$

The final state $\left|\Psi_{\text {final }}\right\rangle$ has an analytic form when $N$ is an even number and can be written as

$$
\begin{aligned}
|\Psi\rangle_{\text {final }}^{I}= & \frac{\sqrt{2}}{4}(-1)^{J} \sum_{m=-J}^{m=J}\left[c_{m}^{J}(\theta)+c_{-m}^{J}(\theta)\right] \\
& \times\left\{\left[1+(-1)^{m}\right]\left(\cos m \phi_{1}+\sin m \phi_{1}\right)\right. \\
& \left.\times-i\left[1-(-1)^{m}\right]\left(\cos m \phi_{1}-\sin m \phi_{1}\right)\right\}|J, m\rangle .
\end{aligned}
$$

Thus, the expectation of the half-population difference and the expectation of the square of the half-population

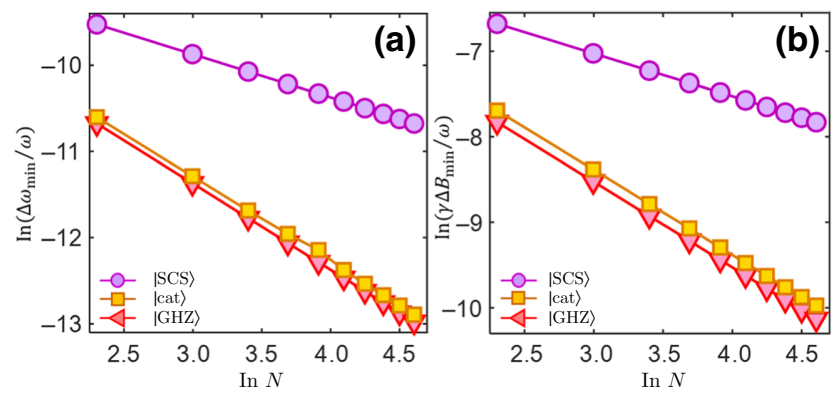

FIG. 14. Log-log plots of the scaling of the optimal measurement precision versus total particle number for (a) frequency $\omega$ and (b) amplitude $B$. The circles show the results for a SCS with $\hat{U}_{I}=e^{-i \frac{\pi}{2} \hat{J}_{y}}$ and the lines are the corresponding fitting curves. The squares show the results for a spin cat state with $\hat{U}_{I}=$ $e^{i \frac{\pi}{2} \hat{J}_{x}} e^{i \frac{\pi}{2} \hat{J}_{z}^{2}} e^{i \frac{\pi}{2} \hat{J}_{x}}$ and $\theta=\pi / 8$ and the lines are the corresponding fitting curves. The triangles show the results for a GHZ state with $\hat{U}_{I}=e^{i \frac{\pi}{2} \hat{J}_{x}} e^{i \frac{\pi}{2} \hat{J}_{z}^{2}} e^{i \frac{\pi}{2} \hat{J}_{x}}$ and the lines are the corresponding fitting curves. Here $T=2 \pi, \omega=20 \pi,\left|\left(\omega-\omega_{e}\right) / \omega\right| \in[0,0.008]$, $\gamma B \in[0,2 \pi]$, and $k=-1$. 
difference are

$$
\begin{aligned}
\left\langle J_{z}\right\rangle_{f} & =(-1)^{L+1} \sum_{m=-J}^{m=J}(-1)^{J-m} m\left|c_{m}^{J}(\theta)\right|^{2} \sin 2 m \phi_{1} \\
\left\langle J_{z}^{2}\right\rangle_{f} & =\sum_{m=-J}^{m=J} m^{2}\left|c_{m}^{J}(\theta)\right|^{2} .
\end{aligned}
$$

The derivative with respect to $\phi_{1}$ reads

$$
\frac{\left\langle d J_{z}\right\rangle_{f}}{d \phi_{1}}=(-1)^{L+1} \sum_{m=-J}^{m=J} m^{2}(-1)^{J-m}\left|c_{m}^{J}(\theta)\right|^{2} \cos 2 m \phi_{1}
$$

Correspondingly, the standard deviation of the halfpopulation difference is given by

$$
\begin{aligned}
\left(\Delta \hat{J}_{z}\right)_{f} & =\sqrt{\left\langle J_{z}^{2}\right\rangle_{f}-\left(\left\langle J_{z}\right\rangle_{f}\right)^{2}} \\
& =\sqrt{\sum_{m=-J}^{m=J} m^{2}\left|c_{m}^{J}(\theta)\right|^{2}-\left[\sum_{m=-J}^{m=J}(-1)^{J-m} m\left|c_{m}^{J}(\theta)\right|^{2} \sin 2 m \phi_{1}\right]^{2}}
\end{aligned}
$$

The measurement signal $J_{z}$ reads

$$
J_{z}=-\sum_{m=-J}^{m=J}(-1)^{J-m} m\left|c_{m}^{J}(\theta)\right|^{2} \sin 2 m \phi_{1}
$$

According to Eq. (J19), the main frequencies of the bisinusoidal oscillation of $J_{z}$ becomes proportional to $2 \mathrm{~m}$.
Furthermore, we find Eq. (J19) with respect to detuning $\omega-\omega_{e}$ is symmetric for fixed $B$ and the lock-in point located at $\omega-\omega_{e}=0$, i.e., $\omega=\omega_{e}$, as shown in Fig. 12 (dashed orange line). Substituting Eqs. (J17) and (J18) into Eqs. (J9) and (J10), one can analytically obtain the measurement precisions $\Delta \omega$ and $\Delta B$. They read

$$
\begin{aligned}
\Delta \omega & =\frac{\tilde{A}_{1} \pi}{2 \gamma B} \frac{\left(\omega-\omega_{e}\right)^{2}}{\sin \left[\left(\omega-\omega_{e}\right) T\right]-T\left(\omega-\omega_{e}\right) \cos \left[\left(\omega-\omega_{e}\right) T\right] \mid}, \\
\Delta B & =\frac{\tilde{A}_{1} \pi}{2 \gamma}\left|\frac{\omega-\omega_{e}}{\sin \left[\left(\omega-\omega_{e}\right) T\right]}\right|
\end{aligned}
$$

where

$$
\tilde{A}_{1}=\frac{\sqrt{\sum_{m=-J}^{m=J} m^{2}\left|c_{m}^{J}(\theta)\right|^{2}-\left[\sum_{m=-J}^{m=J}(-1)^{J-m} m\left|c_{m}^{J}(\theta)\right|^{2} \sin 2 m \phi_{1}\right]^{2}}}{\left.\left|2 \sum_{m=-J}^{m=J} m^{2}(-1)^{J-m}\right| c_{m}^{J}(\theta)\right|^{2} \cos 2 m \phi_{1} \mid}
$$

Particularly, when $\phi_{1}=\pi / 2, \sin 2 m \phi_{1}=0$, and $(-1)^{J-m} \cos 2 m \phi_{1}=1$, the measurement precisions $\Delta \omega$ and $\Delta B$ can be simplified as

$$
\begin{aligned}
\left.\Delta \omega\right|_{\phi_{1}=\pi / 2} & =\frac{\pi}{2 \gamma B} \frac{\sqrt{\sum_{m=-J}^{m=J} m^{2}\left|c_{m}^{J}(\theta)\right|^{2}}}{\left.\left|2 \sum_{m=-J}^{m=J} m^{2}\right| c_{m}^{J}(\theta)\right|^{2} \mid} \frac{\left(\omega-\omega_{e}\right)^{2}}{\left|\sin \left[\left(\omega-\omega_{e}\right) T\right]-T\left(\omega-\omega_{e}\right) \cos \left[\left(\omega-\omega_{e}\right) T\right]\right|} \\
& =C(\theta) \frac{\pi}{2 \gamma B N} \frac{\left(\omega-\omega_{e}\right)^{2}}{\left|\sin \left[\left(\omega-\omega_{e}\right) T\right]-T\left(\omega-\omega_{e}\right) \cos \left[\left(\omega-\omega_{e}\right) T\right]\right|}, \\
\left.\Delta B\right|_{\phi_{1}=\pi / 2} & =\frac{\sqrt{\frac{1}{2} \sum_{m=-J}^{m=J} m^{2}\left|c_{m}^{J}(\theta)\right|^{2}}}{\left.\left|2 \sum_{m=-J}^{m=J} m^{2}\right| c_{m}^{J}(\theta)\right|^{2} \mid} \frac{\pi}{2 \gamma}\left|\frac{\omega-\omega_{e}}{\sin \left[\left(\omega-\omega_{e}\right) T\right]}\right|=C(\theta) \frac{\pi}{2 \gamma N}\left|\frac{\omega-\omega_{e}}{\sin \left[\left(\omega-\omega_{e}\right) T\right] \mid}\right|
\end{aligned}
$$


Here the coefficient $C(\theta)=1+\left[2 \tan ^{2}(\theta / 2) /(1+\right.$ $\left.\left.\tan ^{2}(\theta / 2)\right)\right]$ is independent on the particle number $N$. It is indicated that the measurement precisions $\Delta \omega$ and $\Delta B$ for spin cat states can exhibit Heisenberg-limited scaling. Furthermore, we numerically calculate the optimal measurement precisions versus particle number in the range $\left|\left(\omega-\omega_{e}\right) / \omega\right| \in[0,0.008]$ and for $\gamma B=2 \pi$. According to the fitting results (see Fig. 14), the log-log measurement precisions $\ln \left(\Delta \omega_{\min } / \omega\right) \approx-\ln N-8.26$ and $\ln \left(\gamma \Delta B_{\min } / \omega\right) \approx-\ln N-5.46$. The optimal measurement precision $\Delta \omega_{\min }$ [yellow squares in Fig. 14(a)] and $\Delta B_{\text {min }}$ [yellow squares in Fig. 14(b)] are both beyond SQL scaling and approach Heisenberg-limited scaling. Finally, we also consider using the GHZ state as the input state, i.e., $\theta=0$ in the spin cat state. According to Eqs. (J15) and (J16), the final state $|\Psi\rangle_{\text {final }}^{I}$ reads

$$
\begin{aligned}
|\Psi\rangle_{\text {final }}^{I}= & \frac{1}{\sqrt{2}}\left(\cos J \phi_{1}-\sin J \phi_{1}\right)|J, J\rangle \\
& +\frac{1}{\sqrt{2}}\left(\cos J \phi_{1}+\sin J \phi_{1}\right)|J,-J\rangle .
\end{aligned}
$$

The expectation of the half-population difference and the measurement signal are

$$
\begin{aligned}
\left\langle J_{z}\right\rangle_{f} & =-\frac{N}{2}(-1)^{L} \sin N \phi_{1}, \\
J_{z} & =-\frac{N}{2} \sin N \phi_{1} .
\end{aligned}
$$

Clearly, the main frequencies of the bisinusoidal oscillation of $\left\langle J_{z}\right\rangle_{f}$ become proportional to $N=2 J$. Similarly, we find Eq. (J26) with respect to detuning $\omega-\omega_{e}$ is symmetric for fixed $B$ and the lock-in point located at $\omega-\omega_{e}=0$, i.e., $\omega=\omega_{e}$, as shown in Fig. 12 (blue lines). Moreover, the square of the half-population difference is independent of the two parameters, and becomes $\left\langle J_{z}^{2}\right\rangle_{f}=J^{2}=$ $N^{2} / 4$. According to Eqs. (J9) and (J10), the measurement precisions for $\omega$ and $B$ have an analytic form and they read

$\Delta \omega$

$$
=\frac{\pi}{2 N \gamma B} \frac{\left(\omega-\omega_{e}\right)^{2}}{\left|\sin \left[\left(\omega-\omega_{e}\right) T\right]-T\left(\omega-\omega_{e}\right) \cos \left[\left(\omega-\omega_{e}\right) T\right]\right|},
$$

$$
\Delta B=\frac{\pi}{2 N \gamma}\left|\frac{\omega-\omega_{e}}{\sin \left[\left(\omega-\omega_{e}\right) T\right]}\right|
$$

From Eqs. (J27) and (J28), the measurement precisions $\Delta \omega$ and $\Delta B$ can exhibit Heisenberg-limited scaling. Meanwhile, for fixed $N$, the measurement precision $\Delta \omega$ is dependent on both the detuning $\omega-\omega_{e}$ and $B$, as shown in Fig. 13(c). When $\sin \left[\left(\omega-\omega_{e}\right) T\right]\left(2-\left(\omega-\omega_{e}\right)^{2} T^{2}\right)$
$-2 T\left(\omega-\omega_{e}\right) \cos \left[\left(\omega-\omega_{e}\right) T\right]=0, \Delta \omega$ attains its optimal value. However, the measurement precision $\Delta B$ is dependent only on the detuning $\omega-\omega_{e}$. When it is near resonance $\omega \approx \omega_{e}, \Delta B=\pi / \gamma N T$ is optimal. To confirm the dependence of the measurement precisions on the particle number, we numerically calculate the optimal measurement precisions versus particle number in the range $\left|\left(\omega-\omega_{e}\right) / \omega\right| \in[0,0.008]$ and for $\gamma B=2 \pi$. According to the fitting results (see Fig. 14), the log-log measurement precisions $\ln \left(\Delta \omega_{\min } / \omega\right) \approx-\ln N-8.37$ and $\ln \left(\gamma \Delta B_{\min } / \omega\right) \approx-\ln N-5.53$. For the two parameters, the optimal measurement precisions $\Delta \omega_{\min }$ [red triangles in Fig. 14(a)] and $\Delta B_{\min }$ [red triangles in Fig. 14(b)] both exhibit Heisenberg-limited scaling.

[1] C. W. Helstrom, Quantum Detection and Estimation Theory (Academic Press, New York, 1976).

[2] S. L. Braunstein and C. M. Caves, Statistical Distance and the Geometry of Quantum States, Phys. Rev. Lett. 72, 3439 (1994).

[3] B. M. Escher, R. L. de Matos Filho, and L. Davidovich, General framework for estimating the ultimate precision limit in noisy quantum-enhanced metrology, Nat. Phys. 7, 406 (2011).

[4] R. Demkowicz-Dobrzánski, J. Kołodyński, and M. Gută, The elusive heisenberg limit in quantum-enhanced metrology, Nat. Commun. 3, 1063 (2012).

[5] C. L. Degen, F. Reinhard, and P. Cappellaro, Quantum sensing, Rev. Mod. Phys. 89, 035002 (2017).

[6] M. Vengalattore, J. M. Higbie, S. R. Leslie, J. Guzman, L. E. Sadler, and D. M. Stamper-Kurn, High-Resolution Magnetometry with a Spinor Bose-Einstein Condensate, Phys. Rev. Lett. 98, 200801 (2007).

[7] G. de Lange, Z. H. Wang, D. Ristè, V. V. Dobrovitski, and R. Hanson, Universal dynamical decoupling of a single solid-state spin from a spin Bath, Science 330, 60 (2010).

[8] W. -J. Kuo and D. A. Lidar, Quadratic dynamical decoupling: Universality proof and error analysis, Phys. Rev. A 84, 042329 (2011).

[9] L. Jiang and A. Imambekov, Universal dynamical decoupling of multiqubit states from environment, Phys. Rev. A 84, 060302 (2011).

[10] P. Zanardi, M. G. A. Paris, and L. C. Venuti, Quantum criticality as a resource for quantum estimation, Phys. Rev. A 78, 042105 (2008).

[11] P. C. Maurer, G. Kucsko, C. Latta, L. Jiang, N. Y. Yao, S. D. Bennett, F. Pastawski, D. Hunger, N. Chisholm, M. Markham, D. J. Twitchen, J. I. Cirac, and M. D. Lukin, Room-temperature quantum bit memory exceeding one Second, Science 336, 1283 (2012).

[12] H. Strobel, W. Muessel, D. Linnemann, T. Zibold, D. B. Hume, L. Pezzè, A. Smerzi, and M. K. Oberthaler, Fisher information and entanglement of non-gaussian spin states, Science 345, 424 (2014).

[13] M. Skotiniotis, P. Sekatski, and W. Dür, Quantum metrology for the ising hamiltonian with transverse magnetic field, New J. Phys. 17, 073032 (2015). 
[14] O. Hosten, N. J. Engelsen, R. Krishnakumar, and M. A. Kasevich, Measurement noise 100 times lower than the quantum-projection limit using entangled atoms, Nature 529, 505 (2016).

[15] J. G. Bohnet, B. C. Sawyer, J. W. Britton, M. L. Wall, A. M. Rey, M. Foss-Feig, and J. J. Bollinger, Quantum spin dynamics and entanglement generation with hundreds of trapped ions, Science 352, 1297 (2016).

[16] I. Lovchinsky, A. O. Sushkov, E. Urbach, N. P. de Leon, S. Choi, K. De Greve, R. Evans, R. Gertner, E. Bersin, C. Müller, L. McGuinness, F. Jelezko, R. L. Walsworth, H. Park, and M. D. Lukin, Nuclear magnetic resonance detection and spectroscopy of single proteins using quantum logic, Science 351, 836 (2016).

[17] S. Choi, N. Y. Yao, and M. D. Lukin, Dynamical engineering of interactions in qudit Ensembles, Phys. Rev. Lett, 119, 183603 (2017).

[18] M. J. Biercuk, H. Uys, A. P. VanDevender, N. Shiga, W. M. Itano, and J. J. Bollinger, Experimental uhrig dynamical decoupling using trapped ions, Phys. Rev. A. 79, 062324 (2009).

[19] M. Hirose, C. D. Aiello, and P. Cappellaro, Continuous dynamical decoupling magnetometry, Phys. Rev. A. 86, 062320 (2012).

[20] J. M. Boss, K. S. Cujia, J. Zopes, and C. L. Degen, Quantum sensing with arbitrary frequency resolution, Science 356, 837 (2017).

[21] J. R. Maze, P. L. Stanwix, J. S. Hodges, S. Hong, J. M. Taylor, P. Cappellaro, L. Jiang, M. V. Gurudev Dutt, E. Togan, A. S. Zibrov, A. Yacoby, R. L. Walsworth, and M. D. Lukin, Nanoscale magnetic sensing with an individual electronic spin in diamond, Nature (London) 455, 644 (2008).

[22] G. de Lange, D. Ristè, V. V. Dobrovitski, and R. Hanson, Single-spin magnetometry with multipulse sensing Sequences, Phys. Rev. Lett 106, 080802 (2011).

[23] S. Kotler, N. Akerman1, Y. Glickman, A. Keselman, and R. Ozeri, Single-ion quantum lock-in amplifier, Nature 473, 61 (2011).

[24] R. Shaniv and R. Ozeri, Quantum lock-in force sensing using optical clock doppler velocimetry, Nat Commun. 8, 14157 (2017).

[25] S. Schmitt, T. Gefen, F. M. Stürner, T. Unden, G. Wolff, C. Müller, J. Scheuer, B. Naydenov, M. Markham, S. Pezzagna, J. Meijer, I. Schwarz, M. Plenio, A. Retzker, L. P. McGuinness, and F. Jelezko, Submillihertz magnetic spectroscopy performed with a nanoscale quantum sensor, Science 356, 832 (2017).

[26] K. Shibata, N. Sekiguchi, and T. Hirano, arXiv:2101. $09102 \mathrm{v} 1$.

[27] V. Giovannetti, S. Lloyd, and L. Maccone, quantumenhanced measurements: Beating the standard Quantum Limit, Science 306, 1330 (2004).

[28] V. Giovannetti, S. Lloyd, and L. Maccone, Quantum Metrology, Phys. Rev. Lett. 96, 010401 (2006).

[29] C. Lee, Adiabatic Mach-Zehnder Interferometry on a Quantized Bose-Josephson Junction, Phys. Rev. Lett. 97, 150402 (2006).

[30] V. Giovannetti, S. Lloyd, and L. Maccone, Advances in quantum metrology, Nat. Photonics. 5, 222 (2011).
[31] J. Huang, S. Wu, H. Zhong, and C. Lee, Quantum metrology with cold atoms, Annual Review Cold Atoms Molecules 2, 365 (2014).

[32] C. Lee, Universality and Anomalous Mean-Field Breakdown of Symmetry-Breaking Transitions in a Coupled Two-Component Bose-Einstein Condensate, Phys. Rev. Lett. 102, 070401 (2009).

[33] J. J. Bollinger, W. M. Itano, and D. J. Wineland, Optimal frequency measurements with maximally correlated states, Phys. Rev. A 54, R4649 (1996).

[34] T. Monz, P. Schindler, J. T. Barreiro, M. Chwalla, D. Nigg, W. A. Coish, M. Harlander, W. Hänsel, M. Hennrich, and R. Blat, 14-Qubit Entanglement: Creation and Coherence, Phys. Rev. Lett. 106, 130506 (2011).

[35] L. Pezzé and A. Smerzi, Entanglement, Nonlinear Dynamics, and the Heisenberg Limit, Phys. Rev. Lett. 102, 100401 (2009).

[36] J. Huang, X. Qin, H. Zhong, Y. Ke, and C. Lee, Quantum metrology with spin cat states under dissipation, Sci. Rep. 5, 17894 (2015).

[37] S. D. Huver, C. F. Wildfeuer, and J. P. Dowling, Entangled fock states for robust quantum optical metrology, imaging, and sensing, Phys. Rev. A 78, 063828 (2008).

[38] B. Lücke, M. Scherer, J. Kruse, L. Pezzé, F. Deuretzbacher, P. Hyllus, O. Topic, J. Peise, W. Ertmer, J. Arlt, L. Santos, A. Smerzi, and C. Klempt, Twin matter waves for interferometry beyond the classical Limit, Science 334, 773 (2011)

[39] E. M. Bookjans, C. D. Hamley, and M. S. Chapman, Strong Quantum Spin Correlations Observed in Atomic Spin Mixing, Phys. Rev. Lett. 107, 210406 (2011).

[40] M. Gabbrielli, L. Pezzé, and A. Smerzi, Spin-Mixing Interferometry with Bose-Einstein Condensates, Phys. Rev. Lett. 115, 163002 (2015).

[41] J. L. Helm, T. P. Billam, A. Rakonjac, S. L. Cornish, and S. A. Gardiner, Spin-Orbit-Coupled Interferometry with Ring-Trapped Bose-Einstein Condensates, Phys. Rev. Lett. 120, 063201 (2018).

[42] H. Xing, A. Wang, Q. S. Tan, W. Zhang, and S. Yi, Heisenberg-scaled magnetometer with dipolar spin-1 condensates, Phys. Rev. A 93, 043615 (2016).

[43] J. Huang, M. Zhuang, and C. Lee, Non-gaussian precision metrology via driving through quantum phase transitions, Phys. Rev. A 97, 032116 (2018).

[44] Th. Monz et al., 14-Qubit Entanglement: Creation and Coherence, Phys. Rev. Lett. 106, 130506 (2011).

[45] J. Pan et al., Multiphoton entanglement and interferometry, Rev. Mod. Phys. 84, 777 (2012).

[46] E. Davis, G. Bentsen, and M. Schleier-Smith, Approaching the Heisenberg Limit Without Single-Particle Detection, Phys. Rev. Lett. 116, 053601 (2016).

[47] T. Macrì, A. Smerzi, and L. Pezzè, Loschmidt echo for quantum metrology, Phys. Rev. A 94, 010102 (2016).

[48] F. Fröwis, P. Sekatski, and W. Dür, Detecting Large Quantum Fisher Information with Finite Measurement Precision, Phys. Rev. Lett. 116, 090801 (2016).

[49] S. S. Szigeti, R. J. Lewis-Swan, and S. A. Haine, PumpedUp SU(1,1) Interferometry, Phys. Rev. Lett. 118, 150401 (2017). 
[50] S. P. Nolan, S. S. Szigeti, and S. A. Haine, Optimal and Robust Quantum Metrology Using Interaction-Based Readouts, Phys. Rev. Lett. 119, 193601 (2017).

[51] J. Huang, M. Zhuang, B. Lu, Y. Ke, and C. Lee, Achieving heisenberg-limited metrology with spin cat states via interaction-based readout, Phys. Rev. A 98, 012129 (2018).

[52] O. Hosten, R. Krishnakumar, N. J. Engelsen, and M. A Kasevich, Quantum phase magnification, Science 352, 1552 (2016).

[53] F. Anders, L. Pezzè, A. Smerzi, and C. Klempt, Phase magnification by two-axis countertwisting for detection-noise robust interferometry, Phys. Rev. A 97, 043813 (2018).

[54] D. Linnemann, H. Strobel, W. Muessel, J. Schulz, R. J. Lewis-Swan, K. V. Kheruntsyan, and M. K. Oberthaler, Quantum-Enhanced Sensing Based on Time Reversal of Nonlinear Dynamics, Phys. Rev. Lett. 117, 013001 (2016).

[55] S. C. Burd, R. Srinivas, J. J. Bollinger, A. C. Wilson, D. J. Wineland, D. Leibfried, D. H. Slichter, and D. T. C. Allcock, Quantum amplification of mechanical oscillator motion, Science 364, 1163 (2019).

[56] S. S. Mirkhalaf, S. P. Nolan, and S. A. Haine, Robustifying twist-and-turn entanglement with interaction-based readout, Phys. Rev. A 97, 053618 (2018).

[57] C. W. Helstrom, Minimum mean-squared error of estimates in quantum statistics, Phys. Lett. A 25, 101 (1967).

[58] M. G. A. Paris, QUANTUM ESTIMATION FOR QUANTUM TECHNOLOGY, Int. J. Quantum Inf. 7, 125 (2009).

[59] M. Szczykulska, T. Baumgratz, and A. Datta, Multiparameter quantum metrology, Adv. Phys: X 1, 621 (2016).

[60] G. Ferrini, A. Minguzzi, and F. W. J. Hekking, Number squeezing, quantum fluctuations, and oscillations in mesoscopic bose josephson junctions, Phys. Rev. A 78, 023606 (2008).

[61] G. Ferrini, D. Spehner, A. Minguzzi, and F. W. J. Hekking, Noise in bose josephson junctions: Decoherence and phase relaxation, Phys. Rev. A 82, 033621 (2010).

[62] K. Pawlowski, D. Spehner, A. Minguzzi, and G. Ferrini, Macroscopic superpositions in bose-josephson junctions: Controlling decoherence due to atom losses, Phys. Rev. A 88, 013606 (2013).

[63] Renpeng Fang, Resham Sarkar, and Selim M. Shahriar, Enhancing the sensitivity of an atom interferometer to the heisenberg limit using increased quantum noise, J. Opt. Soc. Am. B 37, 1974 (2020).

[64] A. Van der Ziel, Noise in Measurements (Wiley, Hoboken, NJ, 1976).

[65] P. R. Bevington and D. K. Robinson, Data Reduction and Error Analysis for the Physical Sciences (McGraw-Hill, New York, 1992).

[66] R. Frieden, Probability, Statistical Optics, and Data Testing: A Problem Solving Approach (Springer, Berlin, 2001), 3rd ed., Vol. 10.

[67] R. N. McDonough and A. D. Whalen, Detection of Signals in Noise (Academic Press, San Diego, CA, 1995).

[68] W. Horsthemke and R. Lefever, Noise Induced-Transitions, Theory and Applications in Physics, Chemistry, and Biology (Springer, Berlin, 1984).
[69] H. B. Callen and T. A. Welton, Irreversibility and generalized noise, Phys. Rev. 83, 34 (1951).

[70] Ł. Cywiński, R. M. Lutchyn, C. P. Nave, and S. D. Sarma, How to enhance dephasing time in superconducting qubits, Phys. Rev. B 77, 174509 (2008).

[71] G. Balasubramanian, I. Y. Chan, R. Kolesov, M. AlHmoud, J. Tisler, C. Shin, C. Kim, A. Wojcik, P. R. Hemmer, A. Krueger, T. Hanke, A. Leitenstorfer, R. Bratschitsch, F. Jelezko, and J. Wrachtrup, Nanoscale imaging magnetometry with diamond spins under ambient conditions, Nature 455, 648 (2008).

[72] F. Jelezko, T. Gaebel, I. Popa, M. Domhan, A. Gruber, and J. Wrachtrup, Observation of Coherent Oscillation of a Single Nuclear Spin and Realization of a Two-Qubit Conditional Quantum Gate, Phys. Rev. Lett. 93, 130501 (2004).

[73] C. Gross, T. Zibold, E. Nicklas, J. Estève, and M. K. Oberthaler, Nonlinear atom interferometer surpasses classical precision limit, Nature 464, 1165 (2010).

[74] M. F. Riedel, P. Böhi, Y. Li, T. W. Hänsch, A. Sinatra, and P. Treutlein, Atom-chip-based generation of entanglement for quantum metrology, Nature 464, 1170 (2010).

[75] X. Luo, Y. Zou, L. Wu, Q. Liu, M. Han, M. Tey, and L. You, Deterministic entanglement generation from driving through quantum phase transitions, Science 355, 620 (2017).

[76] Y. Zou, L. Wu, Q. Liu, X. Luo, S. Guo, J. Cao, M. Tey, and L. You, Beating the classical precision limit with spin1 dicke states of more than 10,000 atoms, Proc. Natl. Acad. Sci. 115, 6381 (2018).

[77] S. Choi, N. Y. Yao, and M. D. Lukin, arXiv:1801.00042.

[78] Z. Zhang and L. M. Duan, Generation of Massive Entanglement through an Adiabatic Quantum Phase Transition in a Spinor Condensate, Phys. Rev. Lett. 111, 180401 (2013).

[79] C. F. Ockeloen, R. Schmied, M. F. Riedel, and P. Treutlein, Quantum Metrology with a Scanning Probe Atom Interferometer, Phys. Rev. Lett. 111, 143001 (2013).

[80] W. Muessel, H. Strobel, D. Linnemann, D. B. Hume, and M. K. Oberthaler, Scalable Spin Squeezing for QuantumEnhanced Magnetometry with Bose-Einstein Condensates, Phys. Rev. Lett. 113, 103004 (2014).

[81] S. F. Huelga, C. Macchiavello, T. Pellizzari, A. K. Ekert, M. B. Plenio, and J. I. Cirac, Improvement of Frequency Standards with Quantum Entanglement, Phys. Rev. Lett. 79, 3865 (1997).

[82] L. Pezzè and A. Smerzi, Heisenberg-Limited Noisy Atomic Clock Using a Hybrid Coherent and Squeezed State Protocol, Phys. Rev. Lett. 125, 210503 (2020).

[83] H. T. Ng, Quantum-limited measurement of magnetic-field gradient with entangled atoms, Phys. Rev. A 87, 043602 (2013).

[84] L. Pezzè, A. Smerzi, M. K. Oberthaler, R. Schmied, and P. Treutlein, Quantum metrology with nonclassical states of atomic ensembles, Rev. Mod. Phys. 90, 035005 (2018). 